ARTICLE

https://doi.org/10.1038/s41467-019-13236-9

\title{
Interplay between $\alpha 2$-chimaerin and Rac1 activity determines dynamic maintenance of long-term memory
}

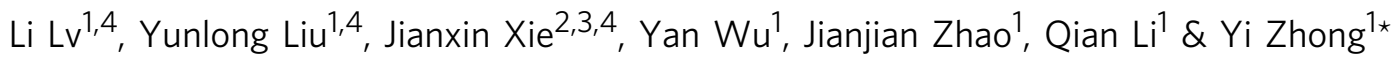

Memory consolidation theory suggests that once memory formation has been completed, memory is maintained at a stable strength and is incapable of further enhancement. However, the current study reveals that even long after formation, contextual fear memory could be further enhanced. Such unexpected enhancement is possible because memory is dynamically maintained at an intermediate level that allows for bidirectional regulation. Here we find that both Rac1 activation and expression of $\alpha 2$-chimaerin are stimulated by single-trial contextual fear conditioning. Such sustained Rac1 activity mediates reversible forgetting, and $\alpha 2-$ chimaerin acts as a memory molecule that reverses forgetting to sustain memory through inhibition of Rac1 activity during the maintenance stage. Therefore, the balance between activated Rac1 and expressed $\alpha 2$-chimaerin defines dynamic long-term memory maintenance. Our findings demonstrate that consolidated memory maintains capacity for bidirectional regulation.

\footnotetext{
${ }_{1}^{1}$ Peking University-Tsinghua University-National Institute Biological Science Joint Graduate Program, Tsinghua-Peking Center for Life Sciences, School of Life Sciences, Tsinghua University, Beijing 100084, China. ${ }^{2}$ National Laboratory of Biomacromolecules, Institute of Biophysics, Chinese Academy of Sciences, 15 Datun Road, Chaoyang District, Beijing 100101, China. ${ }^{3}$ University of Chinese Academy of Sciences, Beijing 100101, China. ${ }^{4}$ These authors contributed equally: Li Lv, Yunlong Liu, Jianxin Xie *email: zhongyithu@tsinghua.edu.cn
} 
M emory consolidation theory suggests that a newly acquired labile memory is gradually consolidated or stabilized through gene-regulation-based persistent modifications in synaptic structures within neural circuits and the resulting consolidated long-term memory (LTM) is maintained at a stable strength ${ }^{1-3}$. Guided by such widely accepted theory, the study of memory maintenance is understandably devoted to the identification of learning-stimulated self-sustainable protein synthesis regulatory mechanisms ${ }^{4,5}$. Indeed, a number of candidate mechanisms for such a simple maintenance have been reported, including the autophosphorylation of calcium/calmodulin-dependent protein-kinase type II (CAMKII) ${ }^{6,7}$, and the persistent protein synthesis regulated via selfperpetuating prion-like translational factors such as the cytoplasmic polyadenylation binding protein-3 (CPEB-3 $)^{8}$. It is a common experience, however, when recalling an episode that happened some time ago, the memory is vivid at one time but vague at others. Such flexible recalling experiences seem to be counterintuitive to memory consolidation theory. In addition to common experiences, it is noteworthy that recent publications have reported a number of intriguing observations that appear inconsistent with the notion of stabilized memory. First, synaptic connections involved in maintaining LTM are highly dynamic ${ }^{5}$. Second, overexpression of the self-autonomous protein-kinaseM-zeta $(\mathrm{PKM} \zeta)^{9}$ was reported to enhance memory long after memory formation, but mechanisms underlying such enhancement remain unexplained ${ }^{10}$.

Forgetting, the flip side of memory consolidation, is also an important feature for different types of memories ${ }^{11}$. The recently proposed concept of active forgetting describes an intrinsic mechanism, i.e., learning or training evokes active forgetting to accelerate the decay of a formed memory. Multiple mechanisms are attributed to forgetting, including through activation of Rac1 or Cdc42, as well as through neurogenesis ${ }^{11}$. Recent progress in study of forgetting reveals that suppression of forgetting, inhibition of Rac1-dependent forgetting in particular, is capable of prolonging an hour-long labile memory to days ${ }^{12-14}$ in a range of tasks from invertebrates to vertebrates ${ }^{15,16}$. Moreover, a study reports that LTM decayed away during infancy can be recovered in adulthood ${ }^{17}$, suggesting that neurogenesis-based forgetting does not lead to memory erasure. These observations got us interested in investigating whether forgetting mechanisms, Rac1dependent forgetting in particular, play any roles in memory maintenance. One possible candidate molecule to inhibit Rac1 activity comes from recent studies in mice during developmental stages $^{18-21}$. It reveals that the negative regulator of Racl activity, a2-chimaerin, acts as a brake through its GTPase-activating function to constrain Rac1 activity in regulating hippocampal dendrite and spine morphogenesis ${ }^{20,22}$ for establishment of normal cognitive ability ${ }^{23}$. However, the roles of interaction between Racl activity and $\alpha 2$-chimaerin in memory maintenance in adulthood remain to be unraveled.

The present study provides a novel mechanism for dynamic memory maintenance that affords an intuitive explanation as to why LTM is still capable of being enhanced or faded long after completing formation. By integrating genetic, pharmacological, and optogenetic manipulations during behavioral paradigms, we demonstrate that the interplay between learning-evoked Rac1dependent forgetting and learning-induced expression of a memory molecule a2-chimaerin enables consolidated contextual fear memory and is maintained at an intermediate level so that memory could be enhanced through expression of more a2chimaerin while attenuated through elevated Racl activity. Moreover, forgetting induced by enhanced Racl activity is reversible through suppression of Rac1 activity. Thus, our results reveal that consolidated memory is maintained through the learning-evoked Rac1-dependent reversible forgetting mechanism balanced by learning-evoked expression of a memory maintenance molecule, a2-chimaerin.

\section{Results}

Inhibition of Rac1 activity causes memory enhancement. As the suppression of active forgetting prolongs hour-long shortterm memory to days ${ }^{12,14}$, we were interested in studying whether Rac1-dependent forgetting mechanisms play roles in LTM maintenance. In an effort to investigate this issue, we assayed Racl activity in hippocampal extracts of mice trained in the contextual fear conditioning (CFC) during the maintenance period of memory. Wild-type (WT) mice were subjected to single- or three-trial CFC and were tested for memory retention at different time points ( $1 \mathrm{~h}$, day 1 , day 7 , day 14 , or day 36) (Fig. 1a and Supplementary Fig. 1a). To avoid the confounding effects of extinction, separate groups of mice were tested at each time point. Consistent with previous reports ${ }^{7}$, mice exposed to three-trial CFC retained a much higher and stable contextual fear memory across the entire testing period (Supplementary Fig. 1a), whereas single-trial CFC produced a protein synthesis-dependent LTM $^{8}$, which was maintained stably for at least 7 days (Fig. 1a) and then decayed from day 7 to day 14, and reached a stable bottom level (dashed line) until day 36 (Fig. 1a). Considering that dynamic memory maintenance, the current work was mainly devoted to single-trial CFC-induced contextual fear memory. Immunoblotting showed that single-trial CFC did not activate Racl initially at $1 \mathrm{~h}$ after training, but significantly increased Racl activity from day 1 to days 2, 7, and 11, and then the activated Racl returned to the basal level on day 14 (Fig. 1b). Consistently, immunofluorescence staining showed about $50 \%$ of hippocampal CA1 cells were labeled with the evoked Racl activation (Fig. 1c and Supplementary Fig. 1b). In addition, neither context nor footshock alone activated Rac1 (Supplementary Fig. 2a). However, three-trial CFC caused the decreased Racl activity (Supplementary Fig. 2d).

The observation in single-trial CFC prompted us to determine whether training-stimulated Racl activity mediates active forgetting of contextual fear memory, as in the case of novel object recognition memory ${ }^{15}$. Mice were bilaterally injected with adenoassociated viruses (AAVs), carrying mutant transgenes that encoded either dominant-negative Racl (Rac1-DN) to inhibit endogenous Racl activity or constitutively active Racl (Racl-CA) to increase Rac1 activity, targeted to hippocampal excitatory neurons ${ }^{15}$ (Supplementary Fig. 3a). Expression of enhanced green fluorescent protein (EGFP) and corresponding changes in Rac1 activity were confirmed (Supplementary Fig. 3b, c). Moreover, such constitutive activation or inhibition of Racl did not cause permanent nonspecific hippocampal damage (Supplementary Fig. 3d). We noted that $1 \mathrm{~h}$ memory after training was similar (Fig. 1d), regardless of whether Rac1 activity was enhanced or inhibited, which is consistent with the notion that Racl activity has no impact on the memory formation ${ }^{12,15,16}$. However, elevated Racl activity accelerated $24 \mathrm{~h}$ memory decay (the bottom level in Fig. 1d), whereas reduced Rac1 activity led to memory enhancement (Fig. 1d), irrespective of memory formation. To corroborate this observation, we also assayed the effects of posttraining inhibition of Rac1 activity through injection of the Rac1 inhibitor Ehop016 ${ }^{15,24,25}$ immediately after training. Reduction of Rac1 activity via Ehop016 injection enhanced memory at $24 \mathrm{~h}$, without affecting memory at $1 \mathrm{~h}$ (Supplementary Fig. 4a, b). Thus, CFC-stimulated Racl activity should accelerate memory decay. Conversely, Rac1 activity inhibition causes memory enhancement. As long-lasting activation of Rac1 following learning is observed in the hippocampus (Fig. 1b, $\mathrm{c}$ and Supplementary 

Contextual fear conditioning
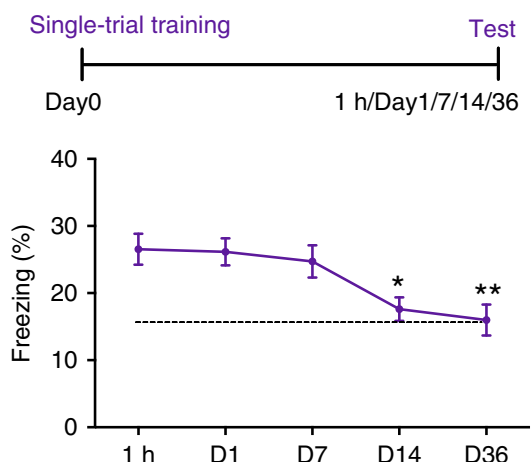

b

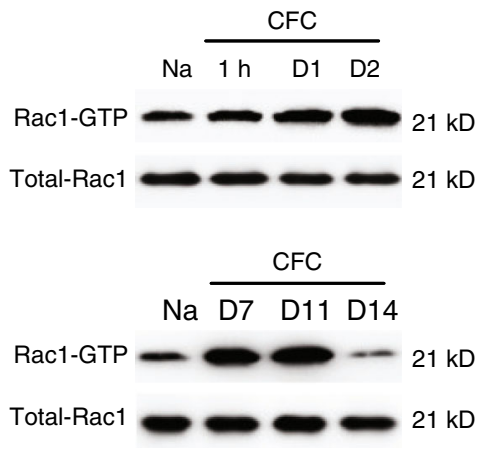

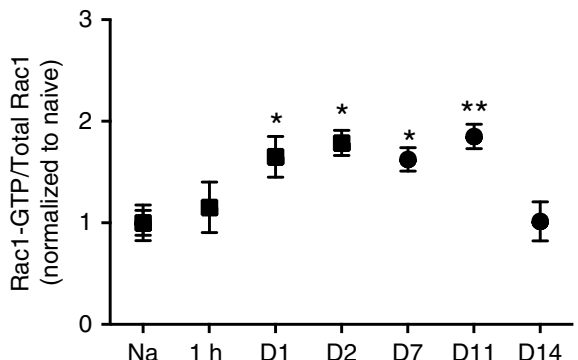

C Rac1 activity in CA1

Rac1-GTP DAPI
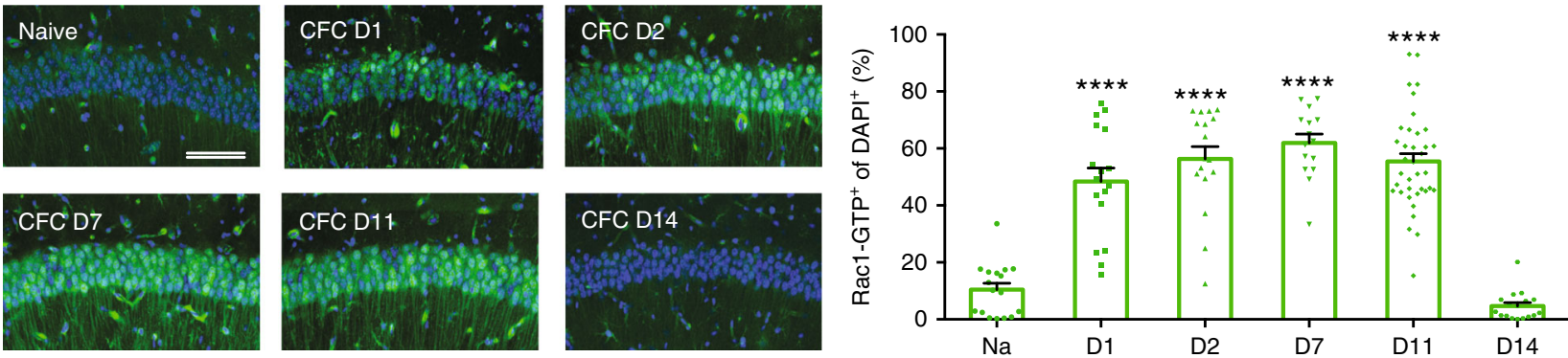

d
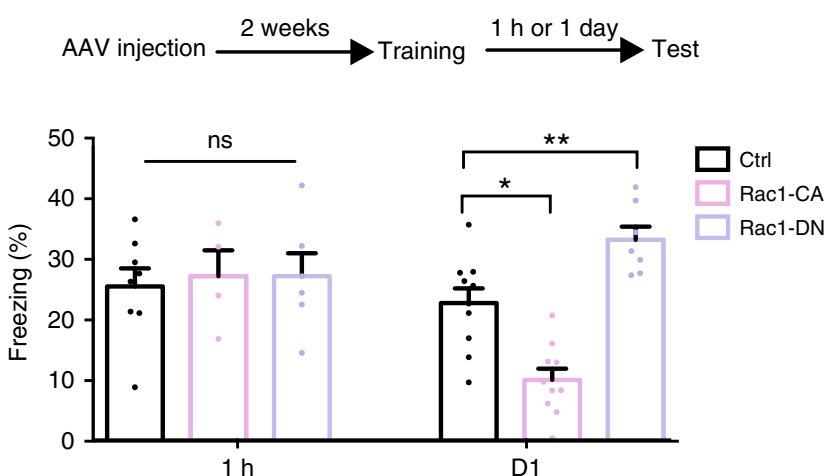

e

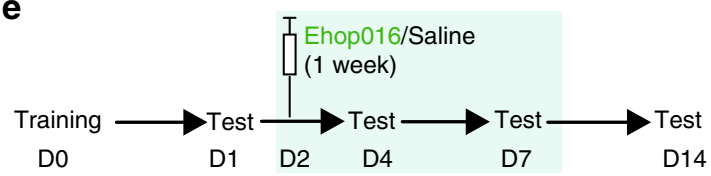

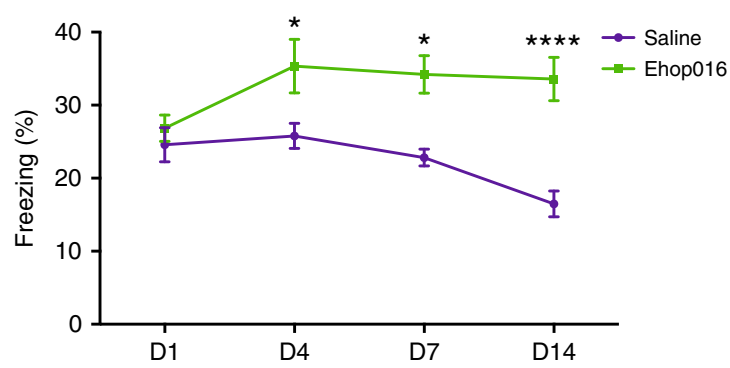

Fig. 1 Inhibition of learning-induced Rac1 activity causes memory enhancement. a Behavioral schedule (top) for contextual fear conditioning (CFC)-induced memory retention curve ( $D$, day) in wild-type (WT) mice (bottom). WT mice were trained using one-shock CFC and then separate group mice were tested for memory retention at various periods ( $1 \mathrm{~h}$, day 1 , day 7 , day 14 , or day 36 ). ${ }^{\star} P<0.05$ and ${ }^{\star \star} P<0.01$ (from one-way ANOVA); $n=41,28,17,22$, and 21 mice. $\mathbf{b}$ Representative image of western blottings (left) and data (right) showing total Rac1 levels and Rac1-GTP in hippocampal extracts from naive (Na) and trained (one shock) at various retention intervals ( $1 \mathrm{~h}$, day 1 , day 2 , day 7 , day 11 , or day 14 ). ${ }^{\star} P<0.05$ and ${ }^{* \star} P<0.01$ (from one-way ANOVA); $n=12$, 7, 7, 3, 4, 4, and 4 mice. c Representative immunostaining of Rac1-GTP (left) and data (right) showing percentages of Rac1-GTP + cells in the hippocampal CA1 region of Na mice and CFC mice at various periods (day 1, day 2, day 7, day 11, or day 14). Coronal sections of the hippocampal CA1 region of Na mice and CFC mice with the anti-Rac1-GTP (green) and anti-nuclei (blue). Scale bar, $500 \mu \mathrm{m}$. ${ }^{\star \star \star \star} P<0.0001$ (from one-way ANOVA); $n=15-17$ slices from 7 mice for each group. $\mathbf{d}$ Behavioral effects of the bidirectional manipulation of hippocampal Rac1 activity on contextual fear memory. After AAV injection for 2 weeks, mice were subjected to one-shock CFC and then separate group mice were tested for 4 min at different time periods ( $1 \mathrm{~h}$ or day 1$)$. ${ }^{\star} P<0.05$ and ${ }^{\star \star} P<0.01$ (from two-way ANOVA); $n=8,10,6,7,4$, and 10 mice. e Time courses of freezing behavior (bottom) in WT mice with injection of Ehop016 or saline (top). ${ }^{\star} P<0.05$ and ${ }^{\star \star \star \star} P<0.0001$ (from two-way ANOVA); $n=10,14$ mice. Data are presented as means \pm SEM. Also see Supplementary Table 1.

Fig. 1b), we hypothesized that memory might be enhanced through the suppression of Racl activity during the maintenance period, even long after memory formation. To further test this hypothesis, we assayed the effects of Racl activity inhibition following the injection of Ehop016 during the maintenance stage. Ehop016 was administered from day 2 after CFC, once per day, for 1 week (Fig. 1e, top), which presumably long after the memory had formed. In this case, multiple injection of Ehop016 did not cause permanent nonspecific hippocampal damage (Supplementary Fig. 4c). In addition, such pharmacological reduction of hippocampal Racl activity had no effects on locomotion or anxiety (Supplementary Fig. 4d, e). Indeed, these 
mice showed normal memory at day 1. Intriguingly, Rac1 pharmacological inhibition enhanced memory in Ehop016treated mice at days 4,7 , and 14 , in comparison with memory in saline-treated mice (Fig. 1e). Moreover, this memory enhancement was retained far beyond the maintenance period and required the continued suppression of Racl activity (Fig. 1e). Normally, the memory decays to a stable bottom level at day 14 (Fig. 1a). Together, inhibition of learning-stimulated hippocampal Racl activity is required for the enhancement of consolidated memory.

Rac1 activity within engram cells affects memory maintenance. Hippocampal CA1 engram cells critically contribute to the maintenance of contextual fear memory ${ }^{26,27}$. We noticed that dorsal hippocampal Rac1 activity was robustly elevated by CFC (Fig. 1b, c and Supplementary Fig. 1b). We thus asked: could this long-lasting activated Racl activity act as a memory suppressor, but not as an eraser within the CA1 engram cells for dynamic memory maintenance? This query leads us to a hypothesis of reversible forgetting-based dynamic memory maintenance that can be described as follows: the memory from day 1 to day 7 is normally maintained at intermediate levels so that bidirectional regulation is permitted.

The following three experiments support this hypothesis. First, we tested whether activation of Racl following learning allocates within the CA1 engram cells. To visualize CFC-activated Rac1 activity within engram cells, we bilaterally injected $A A V_{9}$-c-fos: tetracycline-controlled transactivator ( $\mathrm{tTA}$ ) and $\mathrm{AAV}_{9}$-tetracycline response element (TRE)-Tandem dimer Tomato (tdTomato) to the CA1 region of WT mice (Fig. 2a). Moreover, we examined the viral expression under various treatments, including CFC, context-only, and shock-only (Fig. 2b, c). Upon doxycycline (Dox) withdrawal, engram cells activated by CFC, context-only, or shock-only were labeled with tdTomato $^{28,29}$ (Fig. 2b, c), whereas activated Rac1 under such various treatments in CA1 neurons were labeled with a Rac1-GTP antibody (Fig. 2c and Supplementary Fig. 5a). We observed that CFC, context alone, or shock alone was sufficient to induce tdTomato expression under 2 days off Dox (Fig. 2c and Supplementary Fig. 5a). In particular, the number of $\mathrm{c}^{-} \mathrm{fos}^{+}$cells increased substantially in response to CFC (Fig. 2c, d). Next, we calculated the proportion of double-labeled cells (Fig. 2c and Supplementary Fig. 5a). Immunohistochemistry against activated Racl (Racl$\mathrm{GTP}^{+}$) was assessed by the percentage of $\mathrm{Racl}-\mathrm{GTP}^{+} / \mathrm{c}-\mathrm{fos}^{+}$ colabeled cells among c-fos ${ }^{+}$cells (engram cells). We found that $79 \%$ of ${\mathrm{c}-\mathrm{fos}^{+}}^{+}$cells were labeled with activated Racl positive $\left(\mathrm{Racl}_{-\mathrm{GTP}^{+}}\right)$at $24 \mathrm{~h}$ following training (Fig. 2e). The percentages of double-labeled cells in the group with context alone or shock alone were low (7.4\% and 4.4\%, respectively; Fig. 2e). Moreover, $10 \%$ of non-engram cells were labeled with active Rac1 positive (Supplementary Fig. 5b), demonstrating that learning-specific activated Rac1 allocates within the CA1 engram cells.

Second, we investigated whether memory could be suppressed at any time during the maintenance period through additional activation of Racl within engram cells and such forgotten memory could be salvaged through Racl activity inhibition. The experimental (PaRac1) mice were injected bilaterally into the CA1 with a virus cocktail of $\mathrm{AAV}_{9^{-}}$- -fos:tTA and $\mathrm{AAV}_{9}$-TRE:PaRac1tdTomato to express photoactivatable Rac1 (PaRac1) in CA1 engram cells and the control (tdTomato) mice were injected with $A A V_{9}$-c-fos:tTA and $A A V_{9}$-TRE-tdTomato (Fig. $2 f$, top). In the absence of Dox, training-induced neuronal activity selectively labels active c-fos-expressing CA1 neurons with PaRacl-tdTomato, thereby allowing for the optogenetic activation of Racl at any given time in CA1 engram cells ${ }^{30-32}$. We confirmed that CA1 engram cells were labeled with PaRac1-tdTomato (Fig. 2f, bottom). Activation of Rac1 in CA1 engram cells by blue light (Fig. $2 \mathrm{~g}$ and Supplementary Fig. 6a) at day 4 indeed caused significant and immediate memory decay. However, memory recovered to a normal or intermediate level at day 7 (Fig. 2g). Next, we tested light stimulation-induced PaRacl activation by measuring the level of Racl effector phospho-PAK ${ }^{31}$ in PaRaclexpressing engram cells. Indeed, light stimulation led to increased phospho-PAK in PaRacl-expressing engram cells at day 4 and then such increased phospho-PAK returned to the basal level at day 7 , in comparison with mice that were not subjected to light (Supplementary Fig. 6b-d). Furthermore, optogenetically induced memory decay could also be rapidly restored to an even higher level, through the injection of Ehop016 after light stimulation (Fig. 2h), accompanied with the return of increased p-PAK to the basal level (Supplementary Fig. 6b-d). To further confirm that blue-light-induced memory decay indeed resulted from increased Rac1 activity, Ehop016 was injected prior to light stimulation (Fig. 2i, top). In this case, blue-light exposure was unable to cause memory decay (Fig. 2i, bottom). Moreover, optogenetic stimulation had no impact on locomotion or anxiety (Supplementary Fig. 6e), which are both critical behavioral parameters for the CFC task.

The findings from the above two experiments led us to make a third prediction that the naturally faded memory for several days (staying on day 14; see also Fig. 1a) could be enhanced through the suppression of Racl activity. Injection of the Racl inhibitor for seven consecutive days beginning at day 14 led to recovered memory on day 22 (Fig. 2j). Thus, activation of Racl within engram cells in the CA1 region is sufficient to cause memory decay at any time during the maintenance period. Such optogenetically induced forgotten memory can be rapidly salvaged through Racl pharmacological inhibition, which also recovers naturally faded memory, even several days after the memory had decayed away.

a2-Chimaerin increases following learning are long lasting. If active forgetting is launched following learning, then memories should fade fast ${ }^{12,15,16}$. An obvious question is raised as to how contextual fear memory could be maintained at an intermediate level when facing such sustained Racl-dependent active forgetting (Fig. 1). Subsequent efforts led to the discovery of CFCstimulated expression of a Rac-specific GTPase-activating protein (Rac-GAP), a2-chimaerin, which is known to inactivate Rac1 activity in the nervous system ${ }^{18,19,21}$. Single-trial CFC induced increased expression of a 2 -chimaerin from day 1 to day 7 , but not at $1 \mathrm{~h}$, and this expression returned to the basal level on day 11 (Fig. 3a).

To verify the protein synthesis of a2-chimaerin after singletrial CFC, we utilized a protein synthesis inhibitor anisomycin (ANI). Additional injections of ANI further supported the finding (Fig. 3b). This expression is specific to single-trial CFC but not context alone, shock alone, and three-trial CFC (Supplementary Fig. 2b, e). Furthermore, the expression of its homolog alchimaerin was not stimulated by the CFC (Supplementary Fig. 2c).

a2-Chimaerin interacts with Rac1 to maintain memory. To determine a2-chimaerin functional roles, we manipulated expression levels of $\alpha 2$-chimaerin in the hippocampal CA1 region in which its highest endogenous expression is reported ${ }^{23}$. For knockdown, we generated AAVs that carry a short hairpin RNA (shRNA) sequence ${ }^{22}$ that targets a2-chimaerin (AAVa2 shRNA), fused to tdTomato, under the control of the cytomegalovirus (CMV) early enhancer/chicken $\beta$-actin (CAG) 
a

$$
\begin{aligned}
& \text { c-fos:tTA AAV } \\
& \text { c-fos tTA - } \\
& \text { TRE-dependent AAV }
\end{aligned}
$$

- TRE -tdTomato polyA-

b

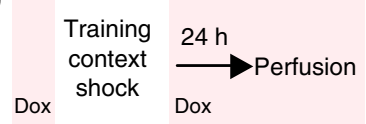

e

CA1 engram labeling with Rac1-GTP

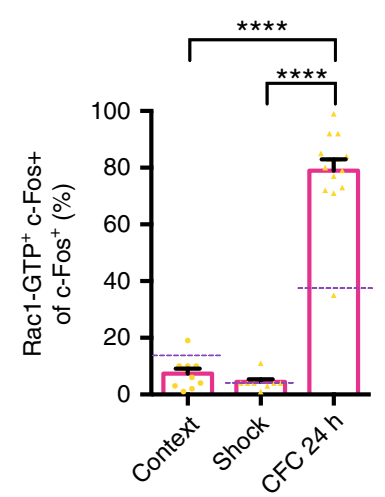

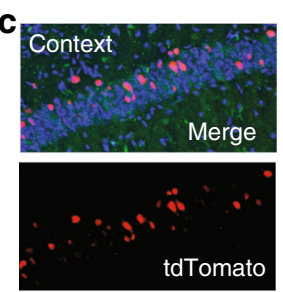
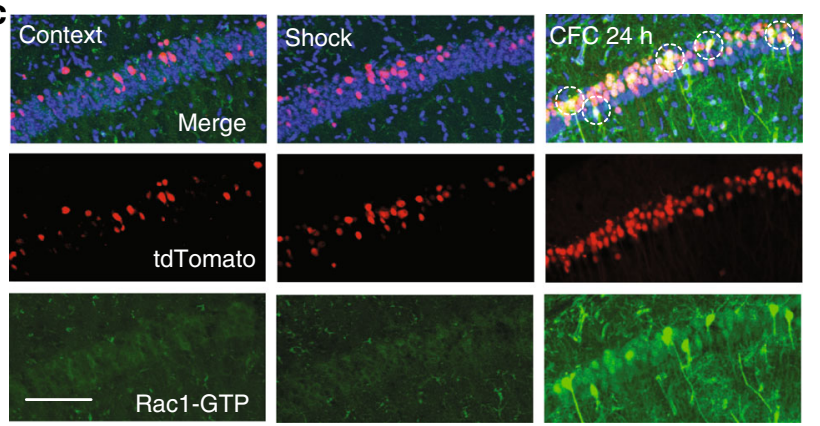

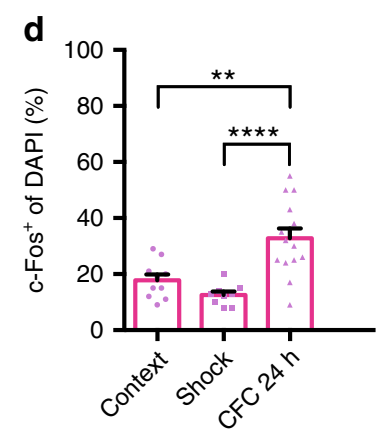

f

CA1 engram manipulation

g c-fos:tTA AAV

$$
\text { - c-fos - tTA - }
$$

TRE-dependent PaRac1 AAV

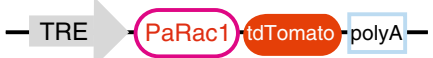

TRE-dependent AAV/Control AAV
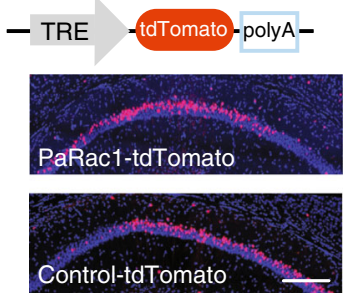

i
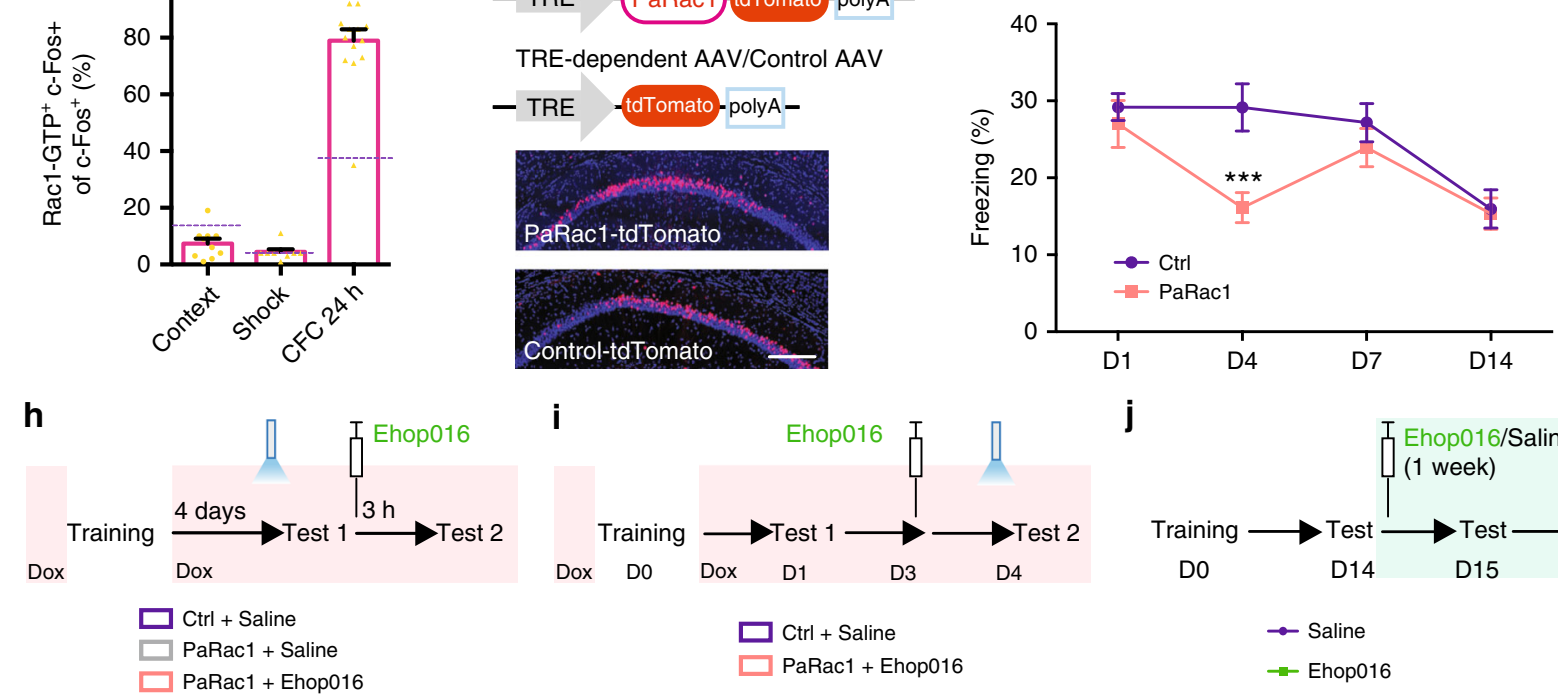

Dox Do
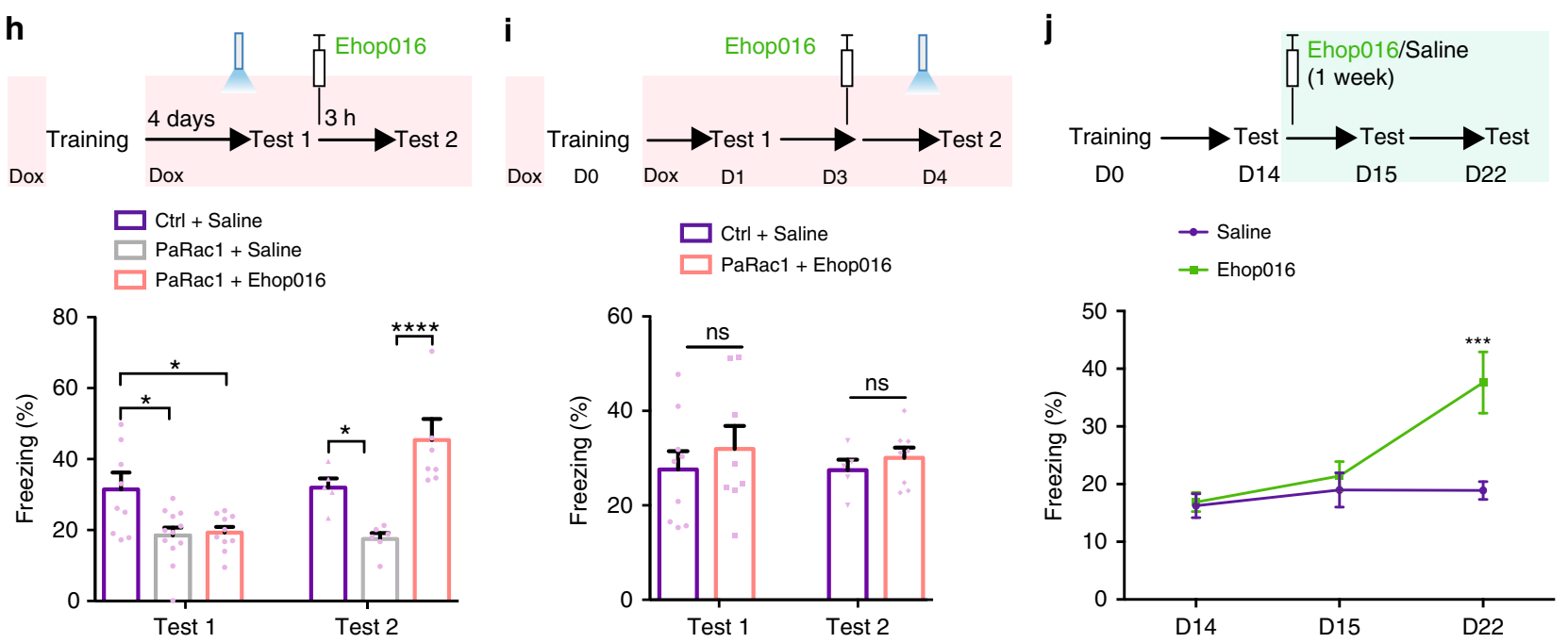

Fig. 2 Regulation of Rac1 activity within CA1 engram cells affects memory maintenance. a-d Strategy of CFC-activated CA1 engram cells labeling with Rac1

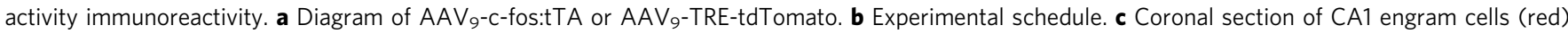
labeling with anti-Rac1-GTP (green). Scale bar, $100 \mu \mathrm{m}$. Circled cells represent examples of double-positive. $\mathbf{d}$ Percentages of engram cells in CA1. ${ }^{\star \star} P<$ 0.01 and ${ }^{\star \star \star \star} P<0.0001$ (from one-way ANOVA); $n=10,9,14$ mice. e Percentages of CA1 engram cells labeling with anti-Rac1-GTP. Chance levels,

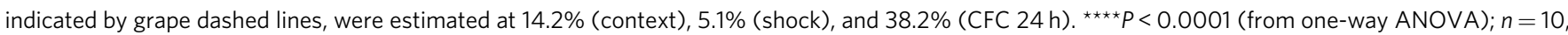

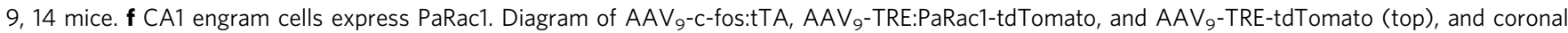
section of the hippocampal CA1 region with PaRac1-tdTomato (red) (bottom). Scale bar, $300 \mu \mathrm{m}$. g-i Experimental schedule (top) and time courses of freezing behavior (bottom). ${ }^{\star \star \star} P<0.001$ (from two-way ANOVA); $n=11,17,15,11,6,12,13,13$ mice (g). $\mathbf{h}$ Test 1 was performed $1.5 \mathrm{~h}$ after light stimulation at day 4. Then, Ehop016 or saline was injected into the mice immediately after Test 1 . Three hours after Ehop016 or saline injection, Test 2 was performed. ${ }^{\star} P<0.05$ and ${ }^{\star \star \star \star} P<0.0001$ (from two-way ANOVA); $n=9,5,10,7,12,6$ mice. i One day after CFC, Test 1 was performed. Two days after Test 1 , mice were injected with Ehop016 or saline. One day later, mice received light stimulation and then Test 2 was performed $1.5 \mathrm{~h}$ after the light stimulation. $n=9,5$, 8, 8 mice (two-way ANOVA). $\mathbf{j}$ Time courses of freezing behavior (bottom) in WT mice with injection of Ehop016 or saline once per day for 1 week (top). ${ }^{\star \star \star} P<0.001$ (from two-way ANOVA); $n=4,4,4,15,11,7$ mice. Data are presented as means \pm SEM. Light red shading signifies that mice are on DOX $(\mathbf{b}, \mathbf{g}, \mathbf{h}, \mathbf{i})$. Data are presented as means \pm SEM. Also see Supplementary Table 1. 
a

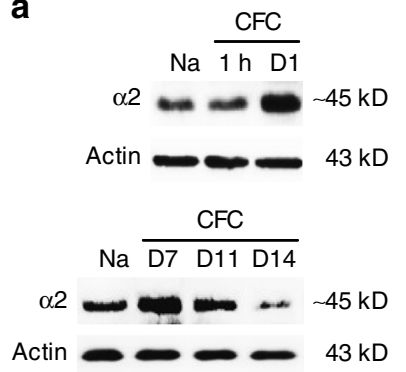

b

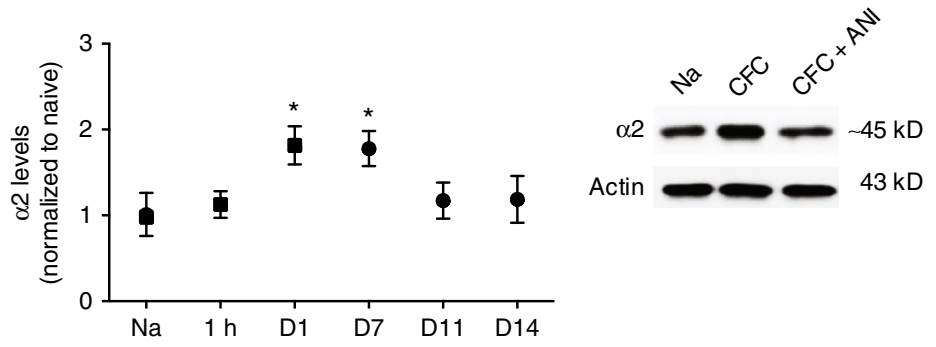

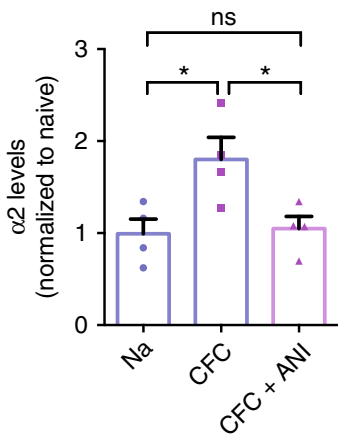

Fig. 3 Learning-evoked the synthesis of Rac-GAP $\alpha 2$-chimaerin. a Representative image of western blottings (left) and data (right) showing hippocampal $\alpha 2$-chimaerin of Na mice and CFC mice. ${ }^{\star} P<0.05$ (from one-way ANOVA); $n=12,4,6,5,6,5$ mice. $\mathbf{b}$ Immunoblotting exhibiting hippocampal $\alpha 2$ chimaerin of Na mice, CFC mice, and CFC mice with ANI treatment (CFC+ANI) mice at 1 day after training. ${ }^{*} P<0.05$ (from one-way ANOVA); $n=4,4,4$ mice. Data are presented as means \pm SEM. Also see Supplementary Table 1.

promoter (Fig. 4a, left). For overexpression, we constructed AAVs that carry an $\alpha 2$-chimaerin encoding gene (AAV- $\alpha 2$-chimaerin), fused to EGFP under the control of the calcium/calmodulindependent protein-kinase IIa (CaMKIIa) promoter (Fig. 4a, middle). Subsequently, mice were injected with either AAV-a2 shRNA or AAV- $\alpha 2$-chimaerin, as well as their controls (AAVscramble shRNA or AAV-control, respectively), in the dorsal hippocampus (Fig. 4a, right). The a2-chimaerin knockdown and overexpression effects were confirmed (Fig. 4b-f), along with the elevated and reduced hippocampal Racl activity, respectively (Fig. 4g, h). In addition, knockdown of a2-chimaerin ( $a 2$ shRNA) enhances the tendency of Racl activity increase following CFC compared with trained (CFC) WT mice (Fig. 4i), validating that partial inhibition of learning-induced Racl activity is indeed a result of a2-chimaerin synthesis. We also found that manipulation of Racl activity had no feedback effect on a2-chimaerin expression (Supplementary Fig. 3e). Moreover, the viral manipulation of $\alpha 2$-chimaerin expression had no impact on locomotion and anxiety (Supplementary Fig. 7a, b).

Knockdown of a2-chimaerin caused rapid memory decay without affecting memory at $1 \mathrm{~h}$. Memory at $24 \mathrm{~h}$ was reduced to a bottom level similar to that in controls at day 14 and remained at that level (Fig. 4j). Conversely, a2-chimaerin overexpression enhanced $24 \mathrm{~h}$ memory to a significantly higher level than that in the ctrl shRNA (ctrl sh) group, which was sustained until day 14 (Fig. 4k). Such a bidirectional memory regulation is resulted from a2-chimaerin-regulated Rac1 activity. Indeed, knockdown of a2chimaerin underlying pharmacological inhibition of Rac1 activity via Ehop016 (Supplementary Fig. 4f) not only failed to reduce memory but, in fact, also greatly enhanced memory from day 1 through day 14 (Fig. 4l). These data, combined with the results in Fig. 1, substantiate that the suppression of Racl activity by the a2-chimaerin synthesis following learning enables the maintenance of an intermediate-level memory state, which allows for dynamic memory maintenance when $\alpha 2$-chimaerin expression is altered.

We also studied the effects of manipulating another member of the $a$-chimaerin family, a1-chimaerin ${ }^{21}$. We found that manipulation of a1-chimaerin expression had no effects on either the memory maintenance induced by single-trial CFC (Supplementary Fig. 8a-e) or on the relevant behavioral parameters (locomotion and anxiety) (Supplementary Fig. 8f). Furthermore, double knockdown of a1-chimaerin and a2-chimaerin had no effects on the relevant behavioral parameters, but yielded an accelerated memory decay phenotype (Supplementary Fig. 9) that is similar to the effects of knockdown of only a2-chimaerin
(Fig. 4j). These data further support a key role of a2-chimaerin, but not a1-chimaerin, in memory maintenance.

Knockdown of a2-chimaerin impairs the multiple types of memories. To examine whether the roles of a2-chimaerin in memory maintenance induced by single-trial CFC could be applied to other hippocampal-dependent associative and reference memory, we carried out other tasks, respectively, including social recognition, novel object recognition, and three-trial CFC. Knockdown of a2-chimaerin caused rapid memory decay of social memory (Fig. 5a) and object recognition memory (Fig. 5b). However, the retention of $24 \mathrm{~h}$ fear memory after three-trial CFC remained normal (Supplementary Fig. 2f). This observation, combined with data relevant to a 2 -chimaerin expression in threetrial CFC (Supplementary Fig. 2e), suggest that a2-chimaerin did not exert effects on memory induced by multiple-trial CFC. Together, these findings identify a2-chimaerin as a memory maintenance molecule in multiple types of hippocampaldependent memories.

Regulation of a2-chimaerin modulates the stability of LTP. To gain further insights into the role of $\alpha 2$-chimaerin in memory enhancement, we recorded long-term potentiation (LTP) induced via a single tetanus (weak stimulation) at the Schaffer collaterals of the hippocampal CA1 region. Knockdown of a2-chimaerin in slices expressing AAV- $\alpha 2$ shRNA led to a reduction of potentiation and accelerated LTP decay (Fig. 6a). In contrast, overexpression of a2-chimaerin in slices expressing AAV-a2chimaerin induced a marked increase of potentiation and a more stable LTP that lasted over $2 \mathrm{~h}$ (Fig. 6b). This observation is consistent with the effects of Racl activity manipulation ${ }^{15}$, even though induction of LTP is slightly changed by knockdown and overexpression of a2-chimaerin. Thus, LTP stability is highly regulated by a2-chimaerin in a pattern similar to memory stability. This role of a2-chimaerin explains why single-trial CFCinduced memory decays from day 7 to day 14 (Fig. 1a), because the expression of $a 2$-chimaerin falls from day 7 to day 11 (Fig. 3a) while activated Rac1 remains at day 11 (Fig. 1b).

\section{Discussion}

The current work put forward the concept of reversible forgetting-based dynamic memory maintenance. Specifically, memory is maintained at an intermediate level, which could be either enhanced or suppressed within a defined range (Fig. 7) during the maintenance stage. Such regulation is mediated by the 
a AAV-mediated knockdown Control virus

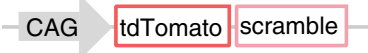

$\alpha 2$-chimaerin shRNA virus

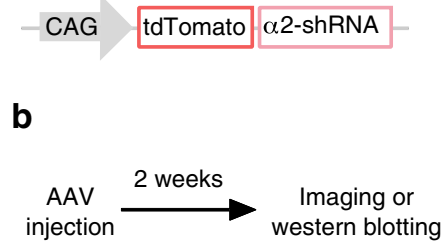

AAV-mediated overexpression

Control virus

CaMKII $\alpha$ EGFP

$\alpha 2$-chimaerin expression virus

CaMKII $\alpha$ $\alpha 2$-chimaerin EGFP

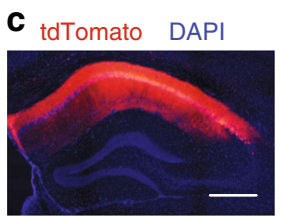

Bilateral AAV injections in hippocampal CA1

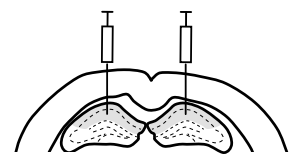

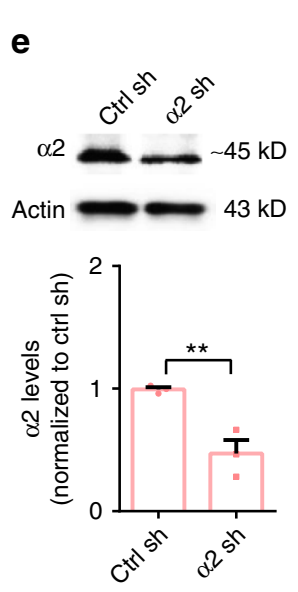

f
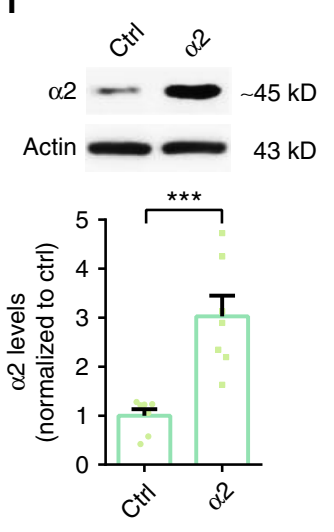

g

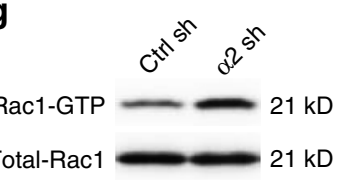

h
(

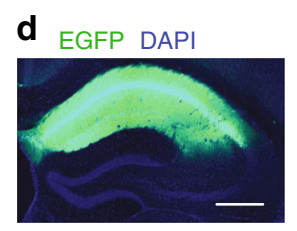

i

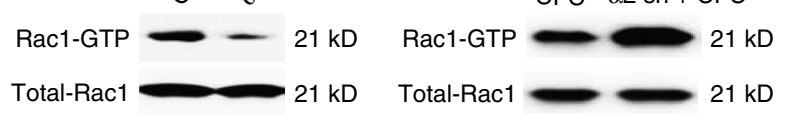

CFC $\alpha 2 \mathrm{sh}+\mathrm{CFC}$
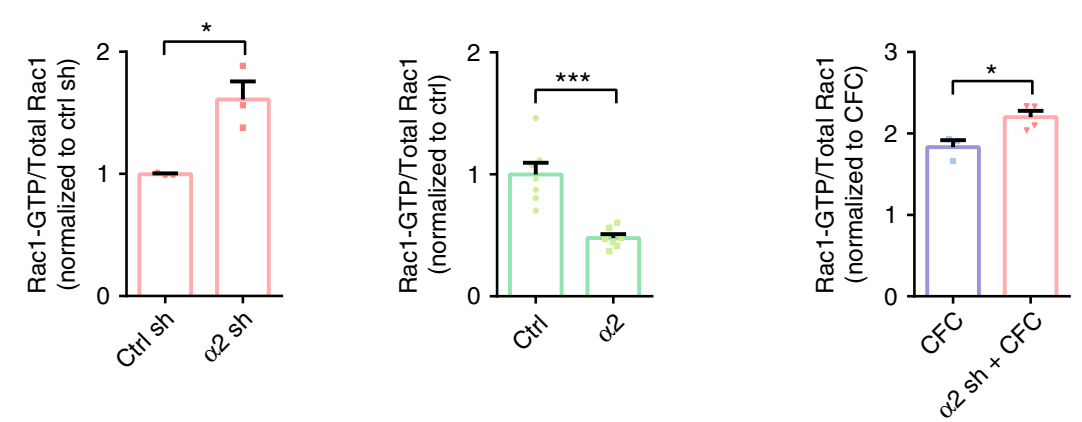

j

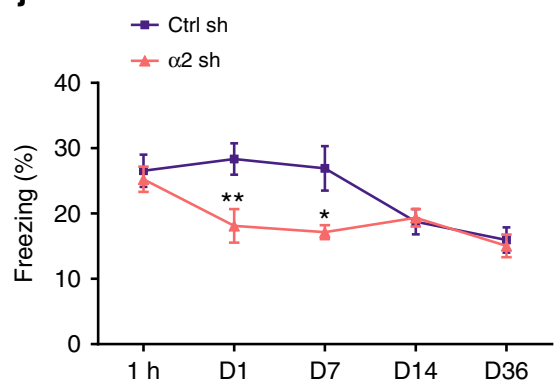

k

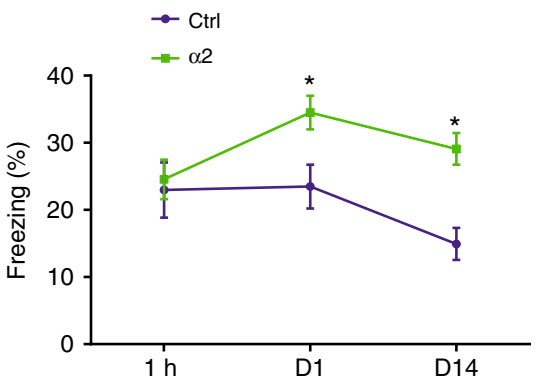

I
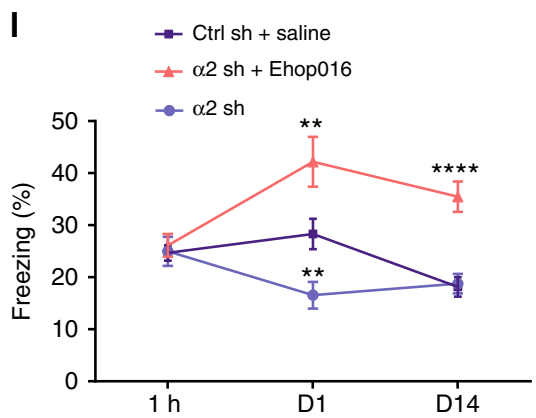

Fig. 4 Interplay between synthesis of $\alpha 2$-chimaerin and Rac1 activity regulates dynamic memory maintenance. a Construction of AAVs and illustration of virus injection sites. b Experimental schedule. c, d Coronal section of the dorsal hippocampus with the viral injections and anti-nuclei (blue). Scale bar, $500 \mu \mathrm{m}$. Bilateral AAV injection in dorsal hippocampus results in specific expression of either tdTomato or EGFP in dorsal hippocampus. e-h Immunoblotting (top) and data (bottom) showing hippocampal $\alpha 2$-chimaerin and Rac1 activity in mice injected with AAV- $\alpha 2$ shRNA ( $\alpha 2$ sh) or AAV$\alpha 2$-chimaerin $(\alpha 2)$, as well as their respective controls. ${ }^{\star} P<0.05,{ }^{\star \star} P<0.01$, and ${ }^{\star \star \star} P<0.001$ (from unpaired $t$-test); $n=3,3$ mice $(\mathbf{e}, \mathbf{g}$ ), $n=7,7$ mice $(\mathbf{f}, \mathbf{h})$. i Immunoblotting (top) and data (bottom) illustrating hippocampal Rac1 activity of CFC mice and trained $\alpha 2$ sh ( $\alpha 2$ sh + CFC) mice at 1 day after training. ${ }^{\star} P<0.05$ (from unpaired $t$-test); $n=3,4$ mice. $\mathbf{j}$-I Time courses of freezing in the ctrl sh (AAV-ctrl shRNA), $\alpha 2$ sh, ctrl (AAV-ctrl), $\alpha 2, c t r l$ sh with saline treatment (ctrl sh + saline), $\alpha 2$ sh with Ehop016 injection ( $\alpha 2$ sh + Ehop016), and $\alpha 2$ sh groups. ${ }^{\star} P<0.05$, ${ }^{\star \star} P<0.01$, and ${ }^{\star \star \star \star} P<0.0001$ (from twoway ANOVA); $n=7,8,15,13,11,11,16,10,9,11$ mice $(\mathbf{j}), n=11,13,11,13,12,7$ mice $(\mathbf{k}), n=3,10,10,8,12,12,5,7,9$ mice $(\mathbf{I})$. Data are presented as means \pm SEM. Also see Supplementary Table 1 .

modulation of reversible Rac1-dependent forgetting or expression of a memory molecule $\alpha 2$-chimaerin. We found that single-trial CFC led to sustained Racl activation from day 1 to day 11 (Fig. 1b, c and Supplementary 1b), which is capable of causing forgetting, as demonstrated by the effects of AAV-mediated expression of constitutively active Racl (Rac1-CA) (Fig. 1d) and optogenetic activation of PaRac1 (Fig. 2g). This forgetting is reversible through inhibition of Racl activity via either injection of a Rac1 inhibitor Ehop016 (Figs. 1e, 2h, j, 4l) or expression of a2-chimaerin (Fig. 4k). The CFC was also capable of stimulating the synthesis of a2-chimaerin that acts as a memory molecule in multiple types of hippocampal-dependent memories through inactivating Racl activity (Figs. 3-5). The role is consistent with its effect in the maintenance of LTP (Fig. 6), as opposing to accelerated decay by Racl activation ${ }^{15}$. Thus, interplay between activated Racl and expressed a2-chimaerin defines the 

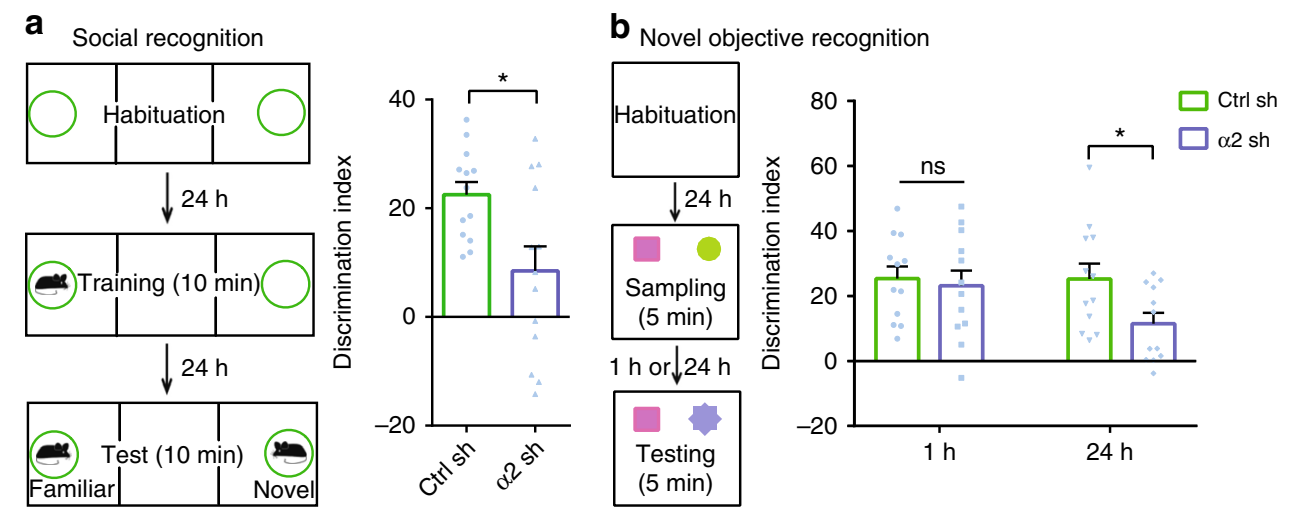

Fig. 5 Knockdown of $\alpha 2$-chimaerin caused rapid decay of social memory and object recognition memory. $\mathbf{a}$, $\mathbf{b}$ Depiction of the experimental design for social recognition and novel object recognition, respectively (left). a Decay of the $24 \mathrm{~h}$ social discrimination memory was induced by AAV- $\alpha 2$ shRNA injection (right). ${ }^{\star} P<0.05$ (from unpaired $t$-test); $n=13,13$ mice. $\mathbf{b}$ Effects of $\alpha 2$-chimaerin knockdown on 1 and 24 h object recognition memory (right). ${ }^{\star} P<0.05$ (from two-way ANOVA), $n=12,12$ mice. Data are presented as means \pm SEM. Also see Supplementary Table 1.
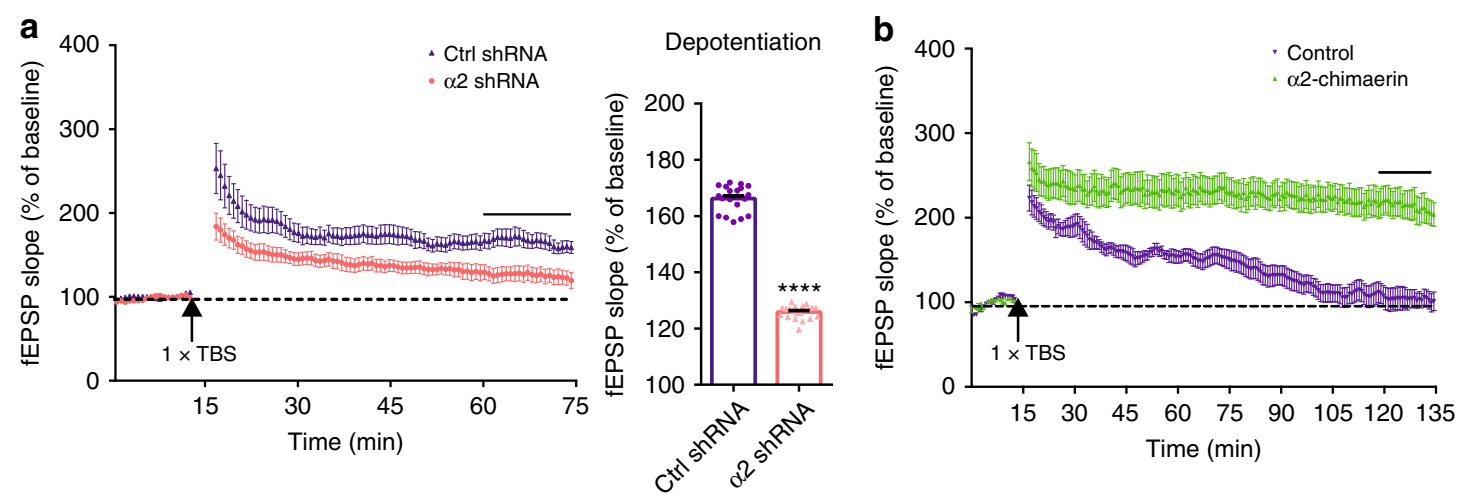

Depotentiation

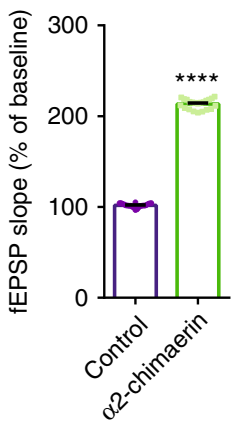

Fig. $6 \alpha 2$-chimaerin knockdown and overexpression modulate the stability of LTP. $\mathbf{a}, \mathbf{b}$ LTP in slices from $\alpha 2$ sh mice and $\alpha 2$ mice, as well as their controls (ctrl sh or ctrl, respectively) (left), and the average fEPSP slope in the last $15 \mathrm{~min}$ of the LTP recording (right). One theta burst stimulation was given 15 min after baseline recording and LTP was recorded in the Schaffer collateral pathway for $1 \mathrm{~h}$ (a) or $2 \mathrm{~h}(\mathbf{b})$. ${ }^{\star \star \star \star} P<0.0001$ (from unpaired $t$-test); $n=8,9$ mice (a), $n=11,8$ mice (b). Data are presented as means \pm SEM. Also see Supplementary Table 1.

intermediate level of memory during maintenance period and allows dynamic memory maintenance (Fig. 7). Taken together, all of the lines of evidence presented support the novel concept of reversible forgetting-based dynamic memory maintenance.

Recent progress has begun to unravel multiple mechanisms underlying active forgetting, including through activation of Rac1 or Cdc42, as well as through neurogenesis ${ }^{11}$. It however remains largely open for cognitive nature and cellular basis of active forgetting, in which whether active forgetting for memory erasure or for impairment of memory retrieval is not undetermined. A recent study of infantile forgetting in mice suggests that neurogenesis-based forgetting does not erase memory, but decreases memory accessibility ${ }^{17}$. Here we showed that Rac1dependent forgetting is also resulting from a decrease in memory accessibility through an increase in Racl activity, while this inaccessibility can be reversed by inhibition of Racl activity via either expression of a2-chimaerin or injection of a Rac1 activity inhibitor (Figs. 1, 2, 4 and 5), suggesting that Rac1-dependent forgetting is reversible in memory trace. This reversible forgetting-based dynamic memory maintenance may not be confined to single-trial CFC-induced contextual fear memory. For the hippocampal-dependent tasks examined, including social recognition (Fig. 5a) ${ }^{16}$ and novel object recognition ${ }^{15}$ (Fig. 5b), memory decay is all regulated through Rac1-dependent active forgetting.
Thus, any experiences that lead to modulation of Racl activity in hippocampus would affect memory accessibility or memory retrieval. Such reversible forgetting-based dynamic memory maintenance might be important for behavior flexibility as well as provide possible mechanisms for symptoms associated with neurological disorders. For instance, study of Alzheimer's disease model suggests that memory loss results from a retrieval problem $^{33}$. It is interesting to investigate whether such a retrieval problem is resulted from enhanced active forgetting.

The enduring efforts in the study of memory maintenance have been in search for molecular mechanisms which permit a LTM to persist ${ }^{5}$. Indeed, there are a number of memory molecules, such as CAMKII, CPEB-3, PKM $\zeta$, and brain-derived neurotrophic factor reported in association with memory maintenance $5,6,8,9,34-36$. Here we show that one-trial CFC leads to long-lasting protein synthesis of a memory molecule a2-chimaerin from day 1 to day 7 (Fig. 3), the duration matches well with memory retention curve. In fact, this work also provides a mechanism for its role in maintaining memory, i.e., through inhibition of Racl-dependent forgetting. Therefore, the memory persists as long as a2-chimaerin expressed.

The translational regulators $\mathrm{CPEB}$ proteins are a family of four paralogs: CPEB-1, 2, 3, and $4^{8,37}$. It is intriguing to note that the CPEB-2 is capable of regulating expression of receptor EphA4 47 , which phosphorylates a2-chimaerin in leading to inhibition of 

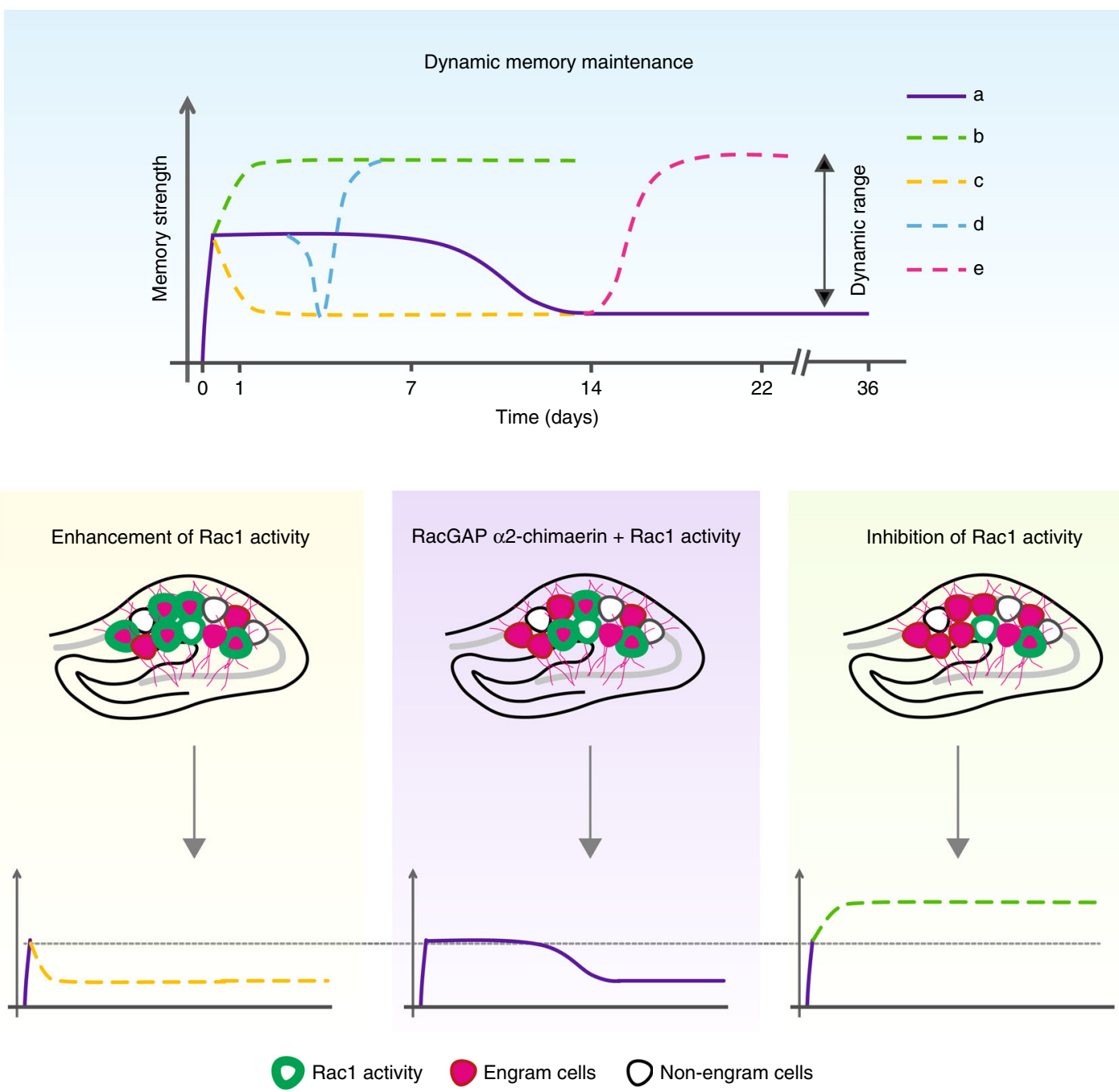

Fig. 7 Model of reversible forgetting-based dynamic memory maintenance. a Intermediate-level memory or normal memory. Memory is maintained at an intermediate level, which is defined by interplay between learning-evoked activated Rac1 and learning-induced expression of $\alpha 2$-chimaerin. $\mathbf{b}$ The top level of dynamic range. Inhibition of Rac1-dependent forgetting enhances consolidated memory through $\alpha 2$-chimaerin overexpression, AAV-mediated expression of dominant-negative Rac1 (Rac1-DN), or injection of a Rac1 inhibitor Ehop016. c The bottom level of dynamic range. Stimulation of Rac1dependent forgetting suppresses memory through $\alpha 2$-chimaerin knockdown, AAV-mediated expression of constitutively active Rac1 (Rac1-CA) or optogenetic activation of PaRac1. d, e Both optogenetically induced and naturally forgotten memory could be recovered through injection of a Rac1 inhibitor.

Rac1 activity $18,19,21$. However, there is no report for how expression of $\alpha 2$-chimaerin is regulated and while CPEB-2 or -3 may regulate its expression 37,38 . It would be of interest to determine long-lasting maintenance of Rac1 activation and a2chimaerin expression induced by learning.

\section{Methods}

Animals. C57BL/6J (age: 3-4 months, male) were purchased from the Vital River Laboratory (Animal Technology, Beijing, China) and were housed in groups under standard conditions according to the Tsinghua University animal facility. Mice were maintained on a $12 \mathrm{~h}$ light/dark cycle and were tested during the light phase of the cycle. All animal work complied with ethical regulations for animal testing and research, and was done in accordance with Institutional Animal Care and Use Committee (IACUC) approval by Tsinghua University and followed by all Association for Assessment and Accreditation of Laboratory Animal Care (AAALAC) guidelines.

DNA constructs. All plasmids were constructed by standard molecular biology procedures and subsequently verified by double-strand DNA sequencing. The a1chimaerin and $\alpha 2$-chimaerin were synthesized by the GENEWIZ company (sequence are showed below). The pAAV-CaMKIIa-EGFP was a gift from Bryan Roth (Addgene plasmid \#50469). The a1-chimaerin and 22 -chimaerin were sub- cloned into AAV backbone pAAV-CaMKIIa-EGFP using the BsrGI/EcoRI restriction enzymes.

al-chimaerin (Sequence ID: NM 001206602.1)

5'-ATGCCATCCAAAGAGTCTTGGTCAGGGAGGAAAACTAATAGGGCT GCAGTTCACAAATCAAAACAAGAGGGCCGTCAGCAAGATTTATTGATAG CAGCCTTGGGAATGAAACTGGGTTCTCCAAAGTCGTCTGTGACAATCTG GCAACCTCTGAAACTCTTTGCTTATTCGCAGTTGACATCACTTGTTAGAA GAGCAACTCTGAAAGAAAACGAGCAAATTCCAAAATATGAAAAGATTCA CAATTTCAAGGTGCATACATTCAGAGGGCCACACTGGTGTGAATACTGT GCCAACTTTATGTGGGGTCTCATTGCTCAGGGAGTGAAATGTGCAGATT GTGGTTTGAATGTTCATAAGCAGTGTTCCAAGATGGTCCCAAATGACTG TAAGCCAGACTTGAAGCATGTCAAAAAGGTCTACAGCTGTGACCTTACG ACGCTCGTGAAAGCACATACCACTAAGCGGCCAATGGTGGTAGACATGT GCATCAGGGAGATTGAGTCTAGAGGTCTTAATTCTGAAGGACTATACCG AGTATCAGGATTTAGTGACCTAATTGAAGATGTCAAGATGGCTTTCGAC AGAGATGGTGAGAAGGCAGATATTTCTGTGAACATGTATGAAGATATCA ACATTATCACTGGTGCACTTAAACTGTACTTCAGGGATTTGCCAATTCCA CTCATTACATATGATGCCTACCCTAAGTTTATAGAATCTGCCAAAATTAT GGATCCGGATGAGCAATTGGAAACCCTTCATGAAGCACTGAAACTACTG CCACCTGCTCACTGCGAAACCCTCCGGTACCTCATGGCACATCTAAAGA GAGTGACCCTCCACGAAAAGGAGAATCTTATGAATGCAGAGAACCTTGG AATCGTCTTTGGACCCACCCTTATGAGATCTCCAGAACTAGACGCCATG GCTGCATTGAATGATATACGGTATCAGAGACTGGTGGTGGAGCTGCTTA TCAAAAACGAAGACATTTTATTTTAA-3

a2-chimaerin (Sequence ID: NM_001822.5) 
5'-ATGGCCCTGACCCTGTTTGATACAGATGAATATAGACCTCCTGTTT GGAAATCTTACTTATATCAGCTACAACAGGAAGCCCCTCATCCTCGAAG AATTACCTGTACTTGCGAGGTGGAAAACAGACCAAAGTATTATGGAAGA GAGTTTCATGGCATGATCTCCAGAGAAGCAGCCGACCAGCTCTTGATTG TGGCTGAGGGGAGCTACCTCATCCGGGAGAGCCAGCGGCAGCCAGGGA CCTACACTTTGGCTTTAAGATTTGGAAGTCAAACCAGAAACTTCAGGCT CTACTACGATGGCAAGCACTTTGTTGGGGAGAAACGCTTTGAGTCCATC CACGATCTGGTGACTGATGGCTTGATTACTCTCTATATTGAAACCAAGGC AGCAGAATACATTGCCAAGATGACGATAAACCCAATTTATGAGCACGTA GGATACACAACCTTAAACAGAGAGCCAGCATACAAAAAACATATGCCAG TCCTGAAAGAGACACATGATGAGAGAGATTCTACAGGCCAGGATGGGG TGTCAGAGAAAAGGTTGACATCACTTGTTAGAAGAGCAACTCTGAAAGA AAACGAGCAAATTCCAAAATATGAAAAGATTCACAATTTCAAGGTGCAT ACATTCAGAGGGCCACACTGGTGTGAATACTGTGCCAACTTTATGTGGG GTCTCATTGCTCAGGGAGTGAAATGTGCAGATTGTGGTTTGAATGTTCA TAAGCAGTGTTCCAAGATGGTCCCAAATGACTGTAAGCCAGACTTGAAG CATGTCAAAAAGGTCTACAGCTGTGACCTTACGACGCTCGTGAAAGCAC ATACCACTAAGCGGCCAATGGTGGTAGACATGTGCATCAGGGAGATTGA GTCTAGAGGTCTTAATTCTGAAGGACTATACCGAGTATCAGGATTTAGT GACCTAATTGAAGATGTCAAGATGGCTTTCGACAGAGATGGTGAGAAGG CAGATATTTCTGTGAACATGTATGAAGATATCAACATTATCACTGGTGCA CTTAAACTGTACTTCAGGGATTTGCCAATTCCACTCATTACATATGATGC CTACCCTAAGTTTATAGAATCTGCCAAAATTATGGATCCGGATGAGCAA TTGGAAACCCTTCATGAAGCACTGAAACTACTGCCACCTGCTCACTGCG AAACCCTCCGGTACCTCATGGCACATCTAAAGAGAGTGACCCTCCACGA AAAGGAGAATCTTATGAATGCAGAGAACCTTGGAATCGTCTTTGGACCC ACCCTTATGAGATCTCCAGAACTAGACGCCATGGCTGCATTGAATGATA TACGGTATCAGAGACTGGTGGTGGAGCTGCTTATCAAAAACGAAGACAT TTTATTTTAA-3

The U6 promoter-driven a1-chimaerin shRNA ( $\alpha 1$ shRNA), $\alpha 2$-chimaerin shRNA ( $\alpha 2$ shRNA), or negative control scrambled shRNA (ctrl shRNA) constructs were synthesized (GENWIZ company) and sub-cloned into AAV backbone pAVV-CAG-tdTomato (Addgene plasmid \# 59462) using the EcoRI/ HindIII and EcoRV/HindIII restriction enzymes, respectively. The al-chimaerin shRNA sequence binding to the mouse $\alpha 1$-chimaerin was $5^{\prime}$-GCTT TCAGCAATGTGTCAT- $3^{\prime 39}$. The $\alpha 2$-chimaerin shRNA sequence binding to the mouse $\alpha 2$-chimaerin was $5^{\prime}$-GCACATGGCAGTCCTGAAA- $3^{\prime}$, whereas the negative control scrambled shRNA (ctrl shRNA) was $5^{\prime}$ -

GTTCATATGTTCACCTATT- ${ }^{\prime 22}$. The U6 promoter sequence was $5^{\prime}$ - GATC CGACGCCGCCATCTCTAGGCCCGCGCCGGCCCCCTCGCACAGACTTGTG GGAGAAGCTCGGCTACTCCCCTGCCCCGGTTAATTTGCATATAATATTT CCTAGTAACTATAGAGGCTTAATGTGCGATAAAAGACAGATAATCTGTT CTTTTTAATACTAGCTACATTTTACATGATAGGCTTGGATTTCTATAAGAGATACAAATACTAAATTATTATTTTAAAAAACAGCACAAAAGGAAACTC ACCCTAACTGTAAAGTAATTGTGTGTTTTGAGACTATAAATATCCCTTGG AGAAAAGCCTTGTTT-3'.

Antibodies and reagents. The following antibodies were used: primary antibodies for a1-chimaerin and $\alpha 2$-chimaerin were raised in rabbits immunized with keyhole limpet hemocyanin-coupled synthetic peptides as previously reported ${ }^{39}$. Keyhole limpet hemocyanin-coupled synthetic peptides for al-chimaerin was

MPSKESWSGRKANR and keyhole limpet hemocyanin-coupled synthetic peptides for a2-chimaerin was HDEKEATGQDGVSEKR. Anti-Racl-GTP (active Rac1) monoclonal antibody was from the NewEast company (Cat. No. 26903). Anti-Rac1 antibody was from BD Transduction Laboratories (Cat. No. 610650). Anti-phospho-PAK1/2/3 antibody was from novusbio company (Cat. No. NB100-82131). Anti-Cleaved Caspase-3 antibody was from Cell Signaling (Cat. No. 9661). Horseradish peroxidase (HRP)-conjugated goat anti-mouse IgG (Cat. No. \#7072) and HRP-conjugated goat anti-rabbit IgG (Cat. No. \#7071) were from Cell Signaling Technology. Alexa Fluro ${ }^{\oplus} 488$ affinipure donkey anti-mouse IgM was from the Jackson ImmunoResearch Laboratories company (Cat. No. \#711-545-140). Actin antibody was from the Merck Millipore company (Cat. No. \#MAB1501).

Drug. Ehop016 (Shanghai Sun-shine Chemical Technology Co., Ltd) was dissolved in a solution containing $1 \%$ dimethylsulfoxide/30\% PEG/1\% Tween-80. Mice were intraperitoneally injected with $20 \mathrm{mg}$ per $\mathrm{kg}$ of Ehop016 solution or an equivalent volume of saline. At the dose used, Ehop016 has high efficiency to block Racl activity $^{15}$. ANI (Selleck, Cat. No. S7409) was dissolved in $0.9 \%$ saline and the $\mathrm{pH}$ was adjusted with $1 \mathrm{~N} \mathrm{HCl}$ to 7.0-7.4. Mice were subcutaneously injected with 150 mg per $\mathrm{kg}$ of ANI or an equivalent volume of saline immediately after training. This amount of ANI was shown to effectively inhibit cerebral protein synthesis in mice $(\sim 96 \%)^{40}$.

Surgery and viral injections. Mice were anesthetized with $0.2 \%$ sodium pentobarbital $(5 \mathrm{ml} / \mathrm{kg})$ and fixed to a stereotaxic frame. Their body temperature was kept at $36^{\circ} \mathrm{C}$ by a heating pad and their skull was exposed. Furthermore, holes in the skull were drilled by a micromotor drill. The injection of the virus was performed using a $10 \mathrm{ml}$ nanofil syringe under controlled by the UMP3 and Micro4 system (WPI), with a speed of $50 \mathrm{nl} / \mathrm{min}$. The dorsal hippocampus injections were bilaterally targeted to $-2.0 \mathrm{~mm} \mathrm{AP,} \pm 1.5 \mathrm{~mm} \mathrm{ML}$, and $-1.5 \mathrm{~mm}$ $\mathrm{DV}$; the DG injections were bilaterally targeted to $-2.0 \mathrm{~mm} \mathrm{AP}, \pm 1.5 \mathrm{~mm} \mathrm{ML}$, and $-2.0 \mathrm{~mm}$ DV. The viral volumes of the AAV-Rac1-CA, AAV-Rac1-DN, and AAVcontrol were $400 \mathrm{nl}$ for DG and $600 \mathrm{nl}$ for CA1. Mice were bilaterally injected with $1 \mu \mathrm{l}$ of either AAV- $\alpha 1$-chimaerin, AAV- $\alpha 2$-chimaerin, AAV-control, AAV- $\alpha 1$ chimaerin shRNA, AAV- $\alpha 2$-chimaerin shRNA, or AAV-control shRNA. For labeling of the $\mathrm{CA} 1$ engram cells, a virus cocktail $\mathrm{AAV}_{9^{-}} \mathrm{c}$-fos:tTA ( $300 \mathrm{nl}$ of $\mathrm{AAV}_{9^{-}}$ c-fos:tTA) and $300 \mathrm{nl}$ of the $\mathrm{AAV}_{9}$-TRE-tdTomato into the CA1 (-2.2 mm AP, $\pm 1.7 \mathrm{~mm} \mathrm{ML}$, and $-1.6 \mathrm{~mm}$ DV). For the activation of Racl activity in CA1 engram cells, a virus cocktail $\mathrm{AAV}_{9}$-c-fos:tTA ( $300 \mathrm{nl}$ of $\mathrm{AAV}_{9}$-c-fos:tTA and 300 $\mathrm{nl}$ of $\mathrm{AAV}_{9}$-TRE:photoactivatable Racl (PaRac1)-tdTomato ${ }^{30,31,41}$ or the AAV9-c fos-EGFP (as the control group) were injected into the mice CA1 (-2.2 mm AP, $\pm 1.8 \mathrm{~mm} \mathrm{ML}$, and $-1.6 \mathrm{~mm} \mathrm{DV}$ ). Following the injections, the needle stayed for $10 \mathrm{~min}$ before slowly withdrawn and the wound sutured. After surgery, animals were allowed to recover for two weeks prior to the performance of all subsequent experiments.

Optical fiber implant. Mice were bilaterally injected with a virus cocktail (500 nl of $\mathrm{AAV}_{9}$-c-fos:tTA) and $500 \mathrm{nl}$ of the $\mathrm{AAV}_{9}$-TRE:PaRac1-tdTomato ${ }^{30,31,41}$ or the AAV9-c-fos-EGFP (as the control group) into the CA1. Furthermore, they were bilaterally implanted with optical fibers ( $200 \mu \mathrm{m}$ core, 0.37 numerical aperture) into the CAl $(-2.2 \mathrm{~mm} \mathrm{AP}, \pm 1.8 \mathrm{~mm} \mathrm{ML}$, and $-1.5 \mathrm{~mm} \mathrm{DV})$. Mice were allowed to recover for two weeks under the ON-Dox condition prior to the start of the behavioral experiments. The cohorts of mice used for the optogenetic engram stimulation, mice were fed regular food, without Dox, for $24-30 \mathrm{~h}$ until contextual fear-conditioning training. After $\mathrm{CFC}$, mice were returned to their home cage under the ON-Dox condition. Following behavioral testing, brain sections were examined to confirm efficient virus-mediated labeling of the target areas.

In vivo photoactivation of Rac1. Mice were intra-CA1 implanted with optic fiber after AAV injection. The optic fibers of mice were connected to a $473 \mathrm{~nm}$ blue laser diode via a FC/PC adaptor. Light intensity $\left(\sim 15 \mathrm{~mW} / \mathrm{mm}^{2}\right)$ was measured at the tip of the fiber. A blue light from a laser was $\mathrm{ON}$ for $30 \mathrm{~s}$ to stimulate the photoactivatable Rac1 for five trials, with trial intervals of $120 \mathrm{~s}^{31}$. For all behavioral procedures, the freezing behavior was measured in light stimulated mice at $1.5 \mathrm{~h}$ after stimulation. Successively, animals were put back to their home cage.

Contextual fear conditioning. The single- and three-trial CFC procedures were based on those of Denny et al. ${ }^{42}$. The fear-conditioning test was conducted using the HABITEST Modular Behavioral Test System. A Coulbourn Habitest chamber $(27 \mathrm{~cm} \times 28 \mathrm{~cm} \times 30.5 \mathrm{~cm})$ had a stainless-steel rod floor which was connected to a shock generator in a sound-attenuating box. In single-trial CFC sessions, mice were individually placed in the conditioning and freely explored the area for $3 \mathrm{~min}$. Then, mice were exposed to one footshock $(2 \mathrm{~s}, 0.8 \mathrm{~mA})$ and were returned to their home cage $30 \mathrm{~s}$ after. In the three-trial CFC task, the footshock $(2 \mathrm{~s}, 0.8 \mathrm{~mA})$ was delivered at 180, 240, and $300 \mathrm{~s}$. Mice remained in the conditioning chamber for a total of $330 \mathrm{~s}$. In only context or only shock training, mice were exposed in a context for 3 min without shock or one footshock $(2 \mathrm{~s}, 0.8 \mathrm{~mA})$ in the absence of context. After that, mice were placed back in their home cage. During testing, mice were placed back in the conditioning chamber for $4 \mathrm{~min}$ at different times $(1 \mathrm{~h}$, days 7,14 , or 36 ). For all procedures, the animal freezing behaviors were monitored using a manufacturer's software.

Social discrimination test in the three-chamber apparatus. Subject mice were individually placed in the three-chamber apparatus for $10 \mathrm{~min}$ to habituate to the environment (day 1). On day 2, a 6-week-old male C57BL/6J mouse was placed in the left grid enclosure for familiarization. Successively, the test mouse was located in the center compartment of the three-chamber apparatus for 10-min social training. During experimental testing ( $1 \mathrm{~h}$ and day 3$)$, a novel 6-week-old male C57BL/6J mouse was placed in the right grid enclosure and the familiar mouse in the left grid enclosure. And then, test mouse was placed into the social chamber and was allowed to explore for $10 \mathrm{~min}$. The amount of time for the test mouse spent in close interaction was recorded using the Any maze system. The discrimination index (DI) was calculated using the following formula: (time exploring the novel mouse - time exploring the familiar mouse)/(time exploring the novel mouse + time exploring the familiar mouse $) * 100$.

Novel object recognition task. For the habituation phase, individual adult male mice were placed in a chamber $(50 \mathrm{~cm} \times 50 \mathrm{~cm} \times 40 \mathrm{~cm})$ and allowed to freely explore the context for $10 \mathrm{~min}$ while being recorded by an overhead camera. This phase was also conducted as the open field test to assess the animal's basal locomotor activity indicated by the average speed and anxiety levels indicated by the percentage of time spent in the middle arena $(30 \mathrm{~cm} \times 30 \mathrm{~cm})$. For the sampling phase, the animal was placed in the same chamber containing two different objects for $5 \mathrm{~min}$ and allowed to explore the object. To saturate the odor left by the previous mice and to make the mice more relaxed, we placed standard animal beddings in the chamber ${ }^{15}$. For the testing phase, one of the objects was exchanged with a new one, and the time spent exploring the two objects was separately recorded using ANY-MAZE software. The DI was calculated using the following 
formula: (time exploring the novel object - time exploring the familiar object)/ (time exploring the novel object + time exploring the familiar object) $\times 100$. For the memory decay curve, time intervals between sampling and testing were 1 and $24 \mathrm{~h}$.

Western blotting. Isolated hippocampi were homogenized in cell lysis buffer (Beyotime, Cat. No. P0013) with protease inhibitors. The protein concentration was determined using the BCA protein assay kit (Beyotime, Cat. No. P0012). For the detection of the levels of a1-chimaerin, a2-chimaerin, total Rac1 and actin, 20 $\mu \mathrm{g}$ of homogenates was separated by $15 \%$ SDS-polyacrylamide gel electrophoresis and transferred to nitrocellulose membranes (Pall Corporation, Cat. No. 66485). Membranes were blocked in milk solution (5\% milk in TBS and $0.1 \%$ Tween 20 ) for one hour at room temperature. Subsequently, membranes were individually incubated with primary antibodies against $\alpha 1$-chimaerin (anti- $\alpha 1$-chimaerin polyclonal antibody, 1:2000, rabbit), a2-chimaerin (anti- $\alpha 2$-chimaerin polyclonal antibody, 1:1000, rabbit), total Racl (1:2000, rabbit), and actin (1:1000, mouse) overnight at $4{ }^{\circ} \mathrm{C}$. All the HRP-conjugated secondary antibodies were used at 1:2000 dilutions for membranes incubation at room temperature for one hour. For the relative level of the Rac-GTP assay, a GST-tagged PAK-PBD beads (Cytoskeleton, Cat. No. \#PAK02) were incubated with the lysate mix $(300 \mu \mathrm{g})$ for overnight at $4{ }^{\circ} \mathrm{C}$. The beads were washed three times with lysis buffer at room temperature. Then, bound proteins were eluted with loading buffer (Beyotime, Cat. No. P0015B) and analyzed by western blotting. Image quantification analysis in bands of the western blots was calculated by the ImageJ software (National Institutes of Health).

Immunofluorescence. Mice were anesthetized with $0.2 \%$ sodium pentobarbital (5 $\mathrm{ml} / \mathrm{kg}$ ) and perfused intracardially with $30 \mathrm{ml}$ of $4 \%$ paraformaldehyde. The brains were fixed in $4 \%$ paraformaldehyde overnight at $4{ }^{\circ} \mathrm{C}$ and serial coronal sections $(30 \mu \mathrm{m})$ were then taken throughout the hippocampus using a vibratome. The floating sections were collected and rinsed in a $0.1 \mathrm{M}$ phosphate-buffered saline (PBS) ( $\mathrm{pH} 7.4$ ) solution with $0.1 \%$ Triton X-100 and incubated with blocking solution ( $10 \%$ donkey serum and $0.1 \%$ Triton $\mathrm{X}-100)$ for $2 \mathrm{~h}$ at room temperature. The slices were then incubated with the following primary antibodies at $4{ }^{\circ} \mathrm{C}$ for overnight: anti-active Rac1 (1:500, mouse), anti-Cleaved Caspase-3 (1:400, rabbit), and anti-phospho-PAK1/2/3 (1:200, rabbit). Following three times rinsed ( $30 \mathrm{~min}$ each) in the PBS solution, slices were incubated with the secondary antibodies conjugated with dyes. Then sections were washed three times with PBS solution and mounted on slides with antifade mounting medium (VECTASHIELD, Cat. No. H-1200). The images of the immunohistochemistry were captured on a Zeiss LSM 710.

Cell counting. Cell counting of c-Fos-positive cells and Racl-active cells was performed in the CA1 by utilizing imaging analysis function of Zeiss software (Zen blue 2.3). The quantification of the number of c-Fos-positive cells was performed by thresholding c-Fos immunoreactivity above background levels automatically. The quantification of the number of Racl-GTP cells was performed by setting the threshold manually from 1000 to 4096 . The quantification of the number of phospho-PAK cells was performed by setting the threshold manually from 692 to 4096. The quantification of the number of cells with 4',6-diamidino-2-phenylindole was performed by setting the threshold manually from 616 to 4096 .

\section{Preparation of the slices and electrophysiological recordings. Transverse} hippocampal slices were prepared from 4-month-old mice. Animals were killed by decapitation in accordance to the institutional regulations. Hippocampi were sliced $(300 \mu \mathrm{m})$ by a VF-300 microtome (Precisionary Instruments) and kept in an interface chamber with oxygenated artificial cerebrospinal fluid (ACSF; $124 \mathrm{mM}$ $\mathrm{NaCl}, 3 \mathrm{mM} \mathrm{KCl}, 1.25 \mathrm{mM} \mathrm{KH}_{2} \mathrm{PO}_{4}, 1 \mathrm{mM} \mathrm{MgSO}, 2 \mathrm{mM} \mathrm{CaCl}_{2}, 26 \mathrm{mM}$ $\mathrm{NaHCO}_{3}$, and $10 \mathrm{mM}$ glucose) for at least $1 \mathrm{~h}$. Following the recovering at room temperature, the slices were transferred to the field excitatory post-synaptic potentials (EPSPs) recording chamber (MED-PG515A, Alpha MED Science) with ACSF (flow rate $1.5 \mathrm{ml} / \mathrm{min}$ ) for at least $20 \mathrm{~min}$ prior to the recording. Filed EPSPs were evoked from CA3-CA1 synapses through the stimulation of the Schaffer collateral axons and recorded in CA1 extracellular fEPSPs. The stimulation intensity was adjusted to elicit a $30 \%$ maximal response. The LTP protocol for the $100 \mathrm{~Hz}$ consisted in $10 \times 100 \mathrm{~Hz}$ bursts (four pulses per burst) with a $200 \mathrm{~ms}$ interval between bursts. Data acquisition and analysis were performed using the multielectrode MED64 hardware and software packages (Panasonic).

Statistics. Statistical analyses were performed in GraphPad Prism. All data were analyzed with an unpaired $t$-test, one-way analysis of variance (ANOVA) or twoway ANOVA where appropriate. The data are shown as the mean \pm SEM and NS indicates non-significance $(p>0.05)$. The significant levels were set to $P=0.05$. Significant for comparison: ${ }^{\star} p<0.05 ;{ }^{* *} p<0.01 ;{ }^{* * *} p<0.001 ;{ }^{* * *} p<0.0001$. The specific statistical tests used and the details of $p$-values for each experiment can be found in Supplementary Table 1.

Reporting summary. Further information on research design is available in the Nature Research Reporting Summary linked to this article.

\section{Data availability}

All pertinent data generated or analyzed during this study are included in this published article and its accompanying Supplementary Information files.

Received: 9 February 2019; Accepted: 22 October 2019; Published online: 22 November 2019

\section{References}

1. Kandel, E. R., Dudai, Y. \& Mayford, M. R. The molecular and systems biology of memory. Cell 157, 163-186 (2014)

2. Squire, L. R., Genzel, L., Wixted, J. T. \& Morris, R. G. Memory consolidation Cold Spring Harb. Perspect. Biol. 7, a021766 (2015).

3. McGaugh, J. L. Memory-a century of consolidation. Science 287, 248-251 (2000)

4. Rayman, J. B. \& Kandel, E. R. Erratum: Functional prions in the brain. Cold Spring Harb. Perspect. Biol. 9, pii: a033597 (2017).

5. Asok, A., Leroy, F., Rayman, J. B. \& Kandel, E. R. Molecular mechanisms of the memory trace. Trends Neurosci. 122, 1473-1490 (2018).

6. Lisman, J. The CaM kinase II hypothesis for the storage of synaptic memory Trends Neurosci. 17, 406-412 (1994).

7. Frankland, P. W., O’Brien, C., Ohno, M., Kirkwood, A. \& Silva, A. J. AlphaCaMKII-dependent plasticity in the cortex is required for permanent memory. Nature 411, 309-313 (2001)

8. Fioriti, L. et al. The persistence of hippocampal-based memory requires protein synthesis mediated by the prion-like protein CPEB3. Neuron $\mathbf{8 6}$ 1433-1448 (2015).

9. Sacktor, T. C. How does PKMzeta maintain long-term memory? Nat. Rev Neurosci. 12, 9-15 (2011).

10. Shema, R. et al. Enhancement of consolidated long-term memory by overexpression of protein kinase Mzeta in the neocortex. Science 331, 1207-1210 (2011)

11. Davis, R. L. \& Zhong, Y. The biology of forgetting-a perspective. Neuron 95 , 490-503 (2017).

12. Shuai, Y. et al. Forgetting is regulated through Rac activity in Drosophila. Cell 140, 579-589 (2010).

13. Zhang, X., Li, Q., Wang, L., Liu, Z. J. \& Zhong, Y. Cdc42-dependent forgetting regulates repetition effect in prolonging memory retention. Cell Rep. 16, 817-825 (2016)

14. Zhang, X., Li, Q., Wang, L., Liu, Z. J. \& Zhong, Y. Active protection: learningactivated Raf/MAPK activity protects labile memory from Racl-independent forgetting. Neuron 98, 142-155 e144 (2018)

15. Liu, Y. et al. Hippocampal activation of Rac1 regulates the forgetting of object recognition memory. Curr. Biol. 26, 2351-2357 (2016).

16. Liu, Y., Lv, L., Wang, L. \& Zhong, Y. Social isolation induces Rac1-dependent forgetting of social memory. Cell Rep. 25, 288-295 e283 (2018).

17. Guskjolen, A. et al. Recovery of "lost" infant memories in mice. Curr. Biol. 28 2283-2290 e2283 (2018).

18. Iwasato, T. et al. Rac-GAP alpha-chimerin regulates motor-circuit formation as a key mediator of EphrinB3/EphA4 forward signaling. Cell 130, 742-753 (2007).

19. Wegmeyer, H. et al. EphA4-dependent axon guidance is mediated by the RacGAP alpha2-chimaerin. Neuron 55, 756-767 (2007).

20. Iwata, R. et al. Developmental RacGAP alpha2-chimaerin signaling is a determinant of the morphological features of dendritic spines in adulthood. $J$. Neurosci. 35, 13728-13744 (2015)

21. Beg, A. A., Sommer, J. E., Martin, J. H. \& Scheiffele, P. alpha2-Chimaerin is an essential EphA4 effector in the assembly of neuronal locomotor circuits. Neuron 55, 768-778 (2007).

22. Valdez, C. M., Murphy, G. G. \& Beg, A. A. The Rac-GAP alpha2-chimaerin regulates hippocampal dendrite and spine morphogenesis. Mol. Cell Neurosci. 75, 14-26 (2016).

23. Iwata, R. et al. RacGAP alpha2-chimaerin function in development adjusts cognitive ability in adulthood. Cell Rep. 8, 1257-1264 (2014).

24. Humphries-Bickley, T. et al. Pharmacokinetics of Rac inhibitor EHop-016 in mice by ultra-performance liquid chromatography tandem mass spectrometry. J. Chromatogr. B Anal. Technol. Biomed. Life Sci. 981-982, 19-26 (2015).

25. Montalvo-Ortiz, B. L. et al. Characterization of EHop-016, novel small molecule inhibitor of Rac GTPase. J. Biol. Chem. 287, 13228-13238 (2012).

26. Ryan, T. J., Roy, D. S., Pignatelli, M., Arons, A. \& Tonegawa, S. Memory Engram cells retain memory under retrograde amnesia. Science 348, 1007-1013 (2015)

27. Goshen, I. et al. Dynamics of retrieval strategies for remote memories. Cell 147, 678-689 (2011).

28. Kitamura, T. et al. Engrams and circuits crucial for systems consolidation of a memory. Science 356, 73-78 (2017).

29. Guo, N. et al. Dentate granule cell recruitment of feedforward inhibition governs engram maintenance and remote memory generalization. Nat. Med. 24, 438-449 (2018). 
30. Wu, Y. I. et al. A genetically encoded photoactivatable Rac controls the motility of living cells. Nature 461, 104-108 (2009).

31. Das, A., Dines, M., Alapin, J. M. \& Lamprecht, R. Affecting long-term fear memory formation through optical control of Racl GTPase and PAK activity in lateral amygdala. Sci. Rep. 7, 13930 (2017).

32. Hayashi-Takagi, A. et al. Labelling and optical erasure of synaptic memory traces in the motor cortex. Nature 525, 333-338 (2015).

33. Roy, D. S. et al. Memory retrieval by activating engram cells in mouse models of early Alzheimer's disease. Nature 531, 508-512 (2016)

34. Sacktor, T. C. \& Hell, J. W. The genetics of PKMzeta and memory maintenance. Sci. Signal. 10, pii: eaao2327 (2017).

35. Dudai, Y. Molecular bases of long-term memories: a question of persistence. Curr. Opin. Neurobiol. 12, 211-216 (2002).

36. Bekinschtein, P. et al. Persistence of long-term memory storage requires a late protein synthesis- and BDNF-dependent phase in the hippocampus. Neuron 53, 261-277 (2007).

37. Turimella, S. L. et al. Characterization of cytoplasmic polyadenylation element binding 2 protein expression and its RNA binding activity. Hippocampus 25, 630-642 (2015).

38. Theis, M., Si, K. \& Kandel, E. R. Two previously undescribed members of the mouse CPEB family of genes and their inducible expression in the principal cell layers of the hippocampus. Proc. Natl Acad. Sci. USA 100, 9602-9607 (2003).

39. Buttery, P. et al. The diacylglycerol-binding protein alphal-chimaerin regulates dendritic morphology. Proc. Natl Acad. Sci. USA 103, 1924-1929 (2006).

40. Davis, H. P. \& Squire, L. R. Protein synthesis and memory: a review. Psychol. Bull. 96, 518-559 (1984).

41. Liao, Z. et al. Fear conditioning downregulates Racl activity in the basolateral amygdala astrocytes to facilitate the formation of fear memory. Front. Mol. Neurosci. 10, 396 (2017).

42. Denny, C. A. et al. Hippocampal memory traces are differentially modulated by experience, time, and adult neurogenesis. Neuron 83, 189-201 (2014).

\section{Acknowledgements}

We greatly thank Professor Peter Scheiffele at University of Basel for providing an anti a2-chimaerin antibody. We thank L.Z. for help with the experiments and all the members of the Zhong lab for their support. This work was supported by grants from the National Science Foundation of China (91632301 to Y.Z.), the Beijing Municipal Science and Technology Commission (Z161100002616010 to Y.Z.) and (Z161100000216132/01 to Y.Z.), the Peking University-Tsinghua University-National Institute Biological Science Joint Graduate Program and the Tsinghua-Peking Joint Center for Life Sciences.

\section{Author contributions}

L.L. and Y.Z. designed the study. L.L., J.X., and Y.L. conducted the viral behavioral experiments. L.L., J.X., and Y.L. performed all electrophysiological experiments. L.L. and Y.W. performed and analyzed all imaging data. L.L., J.Z., and Y.W. performed the optogenetic behavioral experiments. The western blot data were performed and analyzed by L.L. and Q.L. The molecular cloning was conducted by Y.L. The manuscript was written by L.L. and Y.Z.

\section{Competing interests}

The authors declare no competing interests.

\section{Additional information}

Supplementary information is available for this paper at https://doi.org/10.1038/s41467019-13236-9.

Correspondence and requests for materials should be addressed to Y.Z.

Peer review information Nature Communications thanks Takashi Kitamura and the other anonymous, reviewers for their contribution to the peer review of this work. Peer reviewer reports are available.

Reprints and permission information is available at http://www.nature.com/reprints

Publisher's note Springer Nature remains neutral with regard to jurisdictional claims in published maps and institutional affiliations.

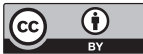

Open Access This article is licensed under a Creative Commons Attribution 4.0 International License, which permits use, sharing, adaptation, distribution and reproduction in any medium or format, as long as you give appropriate credit to the original author(s) and the source, provide a link to the Creative Commons license, and indicate if changes were made. The images or other third party material in this article are included in the article's Creative Commons license, unless indicated otherwise in a credit line to the material. If material is not included in the article's Creative Commons license and your intended use is not permitted by statutory regulation or exceeds the permitted use, you will need to obtain permission directly from the copyright holder. To view a copy of this license, visit http://creativecommons.org/ licenses/by/4.0/.

(C) The Author(s) 2019 